\title{
Color Image Zooming on the Bayer Pattern
}

\author{
Rastislav Lukac, Member, IEEE, Konstantinos N. Plataniotis, Senior Member, IEEE, and \\ Dimitrios Hatzinakos, Senior Member, IEEE
}

\begin{abstract}
A zooming framework suitable for single-sensor digital cameras is introduced and analyzed in this paper. The proposed framework is capable of zooming and enlarging data acquired by single-sensor cameras that employ the Bayer pattern as a color filter array (CFA). The approach allows for operations on noise-free data at the hardware level. Complexity and cost implementation are thus greatly reduced. The proposed zooming framework employs: 1) a spectral model to preserve spectral characteristics of the enlarged CFA image and 2) an adaptive edge-sensing mechanism capable of tracking the underlying structural content of the Bayer data. The framework readably unifies numerous solutions which differ in design characteristics, computational efficiency, and performance. Simulation studies indicate that the new zooming approach produces sharp, visually pleasing outputs and it yields excellent performance, in terms of both subjective and objective image quality measures.
\end{abstract}

Index Terms-Bayer pattern, color filter array (CFA), demosaicking, digital still camera, image restoration, interpolation, zooming.

\section{INTRODUCTION}

$\mathbf{T}$ HE commercial proliferation of single-sensor digital cameras (Fig. 1) ${ }^{1}$ and their use in novel applications, such as wireless phones, sensor networks, pocket devices, surveillance, and automotive apparatus, have increased the demand for new algorithmic and technical developments in the area of color filter array (CFA) image filtering [1], [2], demosaicking [3]-[7], postprocessing [8], compression [9]-[11], and zooming [12]-[14]. Most solutions are designed to operate on the Bayer pattern ${ }^{2}$ which provides an array or mosaic of red $(\mathrm{R})$, green $(\mathrm{G})$ and blue (B) color components (Fig. 2) [17]. Since only a single color component is available at each spatial location, the so-called CFA interpolation [3], [18], [19] or demosaicking [4], [5], [20] process is employed in the imaging pipeline to produce the full color (RGB) camera output.

The camera acquires the scene by first (Fig. 1) focusing and transmitting the light through the Bayer CFA and then sampling the visual information using the charge-coupled device (CCD) [21], [22] or complementary metal-oxide semiconductor

Manuscript received October 3, 2003; revised August 17, 2004. The work of R. Lukac was supported in part by a NATO/NSERC Science Award. This paper was recommended by Associate Editor M. G. Strintzis.

The authors are with the Multimedia Laboratory, The Edward S. Rogers, Sr., Department of Electrical and Computer Engineering, University of Toronto, Toronto, ON M5S 3G4, Canada (e-mail: lukacr@ieee.org).

Digital Object Identifier 10.1109/TCSVT.2005.856923

\footnotetext{
${ }^{1}$ The figures in this paper can be seen in color at http://www.dsp.utoronto.ca/ lukacr/color3.pdf.

${ }^{2}$ By allocating more spatial samples (50\%) to the Green plane, the Bayer pattern improves the perceived sharpness of the digital image [3] since it is well known that the human visual system (HVS) is more sensitive to luminance which is composed primarily of green light [15], [16].
}

(CMOS) [23], [24] sensor, ${ }^{3}$ and an analog-to-digital (A/D) converter. The DRAM buffer temporally stores the digital data from the A/D converter and then passes them to the application-specific integrated circuit (ASIC) which realizes the digital data processing operations such as demosaicking [25], [26]. The demosaicking step [Fig. 3(a)] essentially performs spectral interpolation since it [1], [8], [27]: 1) re-arranges the acquired grayscale (scalar) sensor data to the RGB vectorial field; 2) completes missing color components from the adjacent Bayer data using an interpolator operating in the spectral (color) domain, and 3) preserves the dimensions (spatial resolution) of the input image by generating an output image with spatial dimensions identical to those of the input.

Since the cost of digital cameras rapidly increases with optical zooming and Megapixel capturing capabilities, to keep it at a reasonable level, camera manufacturers produce cameras capable of performing digital zooming. An image zooming technique [Fig. 3(b)] performs spatial interpolation since it [1], [28], [29]: 1) enlarges the spatial resolution of the input image; 2) completes the missing data from the spatially adjacent data using some type of the interpolation operations; and 3) preserves the spectral representation of the input image. In other words, a zooming solution operating on a gray-scale image, such as CFA image, generates a gray-scale image of different spatial dimensions but not a color image. As can be seen from the above listings and the example shown in Fig. 3, the demosaicking and zooming processing steps are fundamentally different in terms of their functionality, although they often employ similar, if not identical, signal processing concepts. Finally, it should be noted that both demosaicking and zooming operations are probably the most commonly performed processing operations in digital cameras.

In this paper, a unique edge-sensing CFA zooming framework for single-sensor imaging devices is introduced. Instead of replicating CFA pixels as was done in [12], [30], the new method generalizes the proposal of [14] and assigns original CFA pixels to positions in an enlarged CFA image using, in a systematic way, nonlinear, edge-sensing spatial interpolators. Unlike the approach of [14], the proposed solution constitutes a powerful framework that unifies previously presented results and ensures compatibility with demosaicking schemes such as those listed in [3], [4], [18], [19], and [31]. In particular, by employing the spectral modeling approaches of [8], [27], [32], and [33], the proposed zooming framework incorporates the spectral image characteristics into the spatial interpolation process to enhance performance. To eliminate the blurred details caused by

\footnotetext{
${ }^{3}$ Since the sensor is essentially a monochromatic device, the raw sensor data captured by a single-sensor camera constitutes a gray-scale image [4], hereafter called the CFA image.
} 


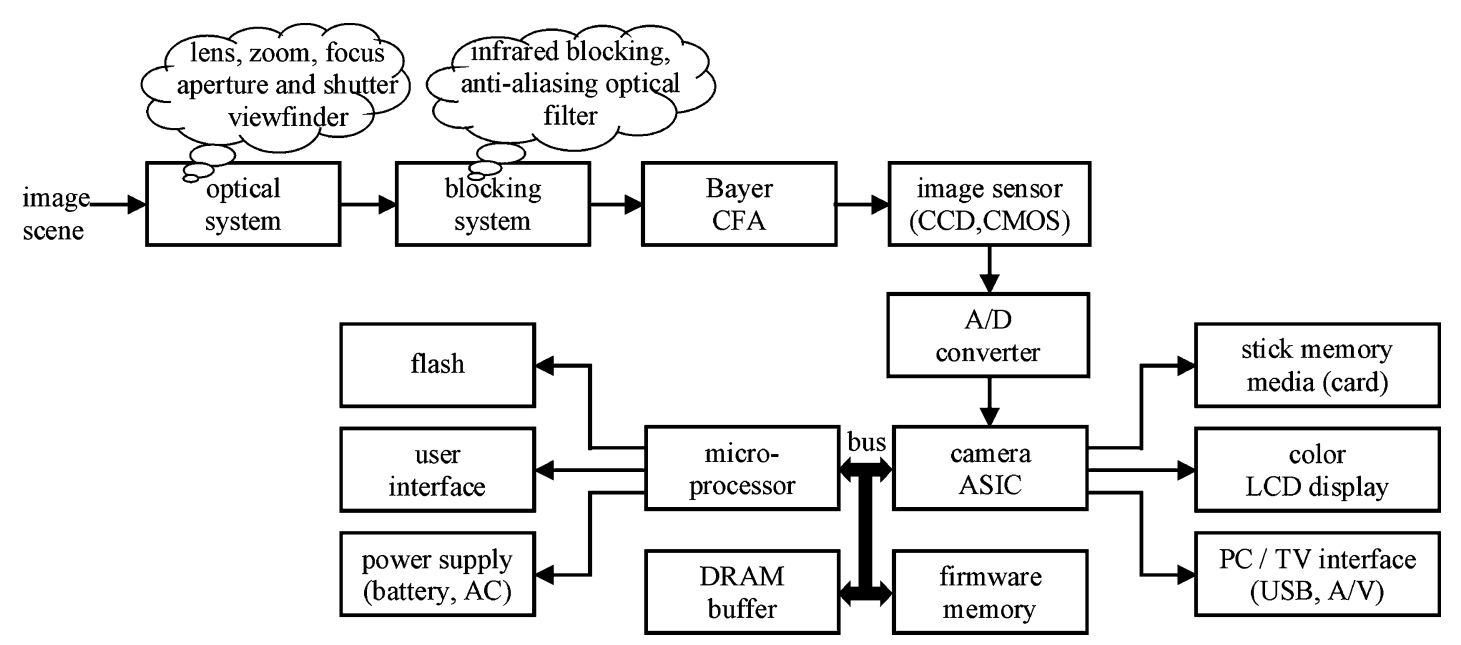

Fig. 1. Hardware components used in a conventional single-sensor digital camera.

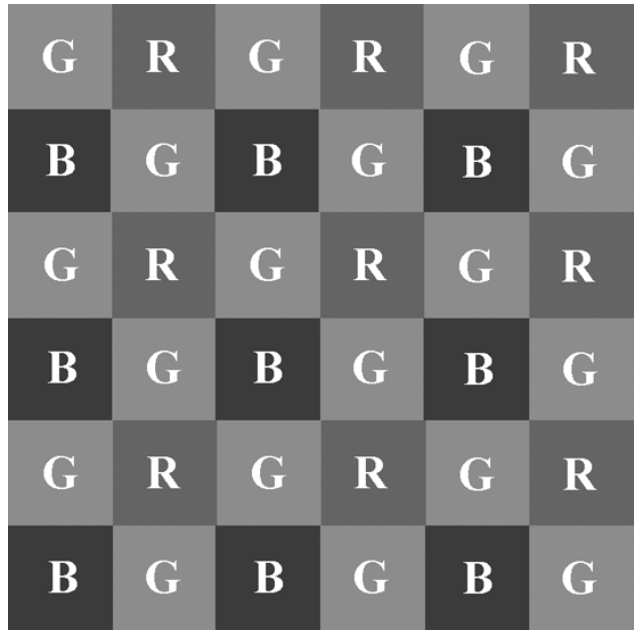

Fig. 2. RGB Bayer CFA pattern [17] with the GRGR phase in the first row. In this CFA, green color filters are arranged in a quincunx lattice format, whereas red and blue color filters are arranged in a rectangular lattice format.

the lack of edge sensitivity in the nonadaptive linear interpolation approach of [14], the proposed framework utilizes a variety of edge-sensing mechanisms. Thus, the missing CFA data in the spatially enlarged Bayer image are interpolated using spectral, spatial, and structural information. The nonlinear, data-adaptive nature of the solution sets it apart from all previously proposed CFA zooming approaches including the linear solution of [14]. To the best of the authors' knowledge, the approach proposed here is the first attempt to devise an adaptive, spectral modeling-based zoomer. Results included in this paper show that the proposed solution produces sharp, visually pleasing color outputs with excellent fidelity in both color and structural content.

The remainder of this paper is organized as follows. The state-of-the-art image zooming techniques are briefly described in Section II. The proposed method is introduced in Section III. Motivation and design characteristics are discussed in detail. In Section IV, the proposed framework is tested using a variety of images. Performance comparisons with relevant zooming approaches are provided. Evaluations are performed in terms of commonly used image quality measures both subjectively

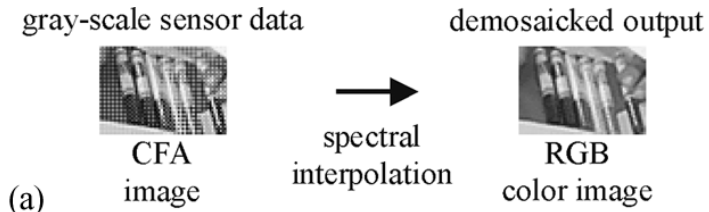

enlarged CFA (gray-scale) image

CFA (gray-scale) image
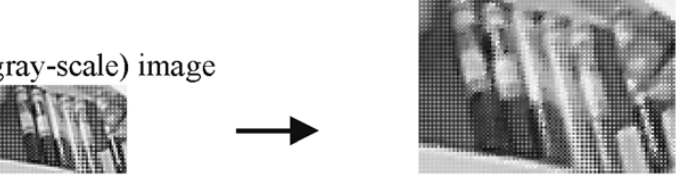

(b)

spatial

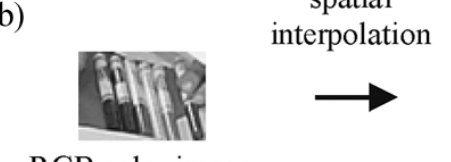

RGB color image

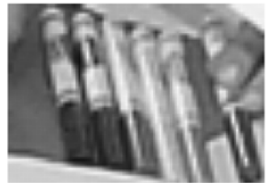

enlarged RGB color image

Fig. 3. Main differences between demosaicking and zooming operations: (a) demosaicking transforms a gray-scale (scalar) CFA-sensor image to a full color (vector) image of the same spatial dimensions while (b) zooming transforms a gray-scale image to an enlarged gray-scale image, or it transforms a color image to an enlarged color image.

and objectively. The computational complexity of the proposed method is analyzed in Section V. Finally, this paper concludes in Section VI.

\section{PRIOR ART}

When single-sensor digital cameras are used, the spatial resolution of the acquired, displayed, and transmitted color images can be increased in two ways, namely as follows:

- demosaicked image zooming [Fig. 4(a)], which employs a demosaicking scheme at the first processing stage to produce a full color image. The spatial resolution of the demosaicked image is then increased using a color image 
(a)

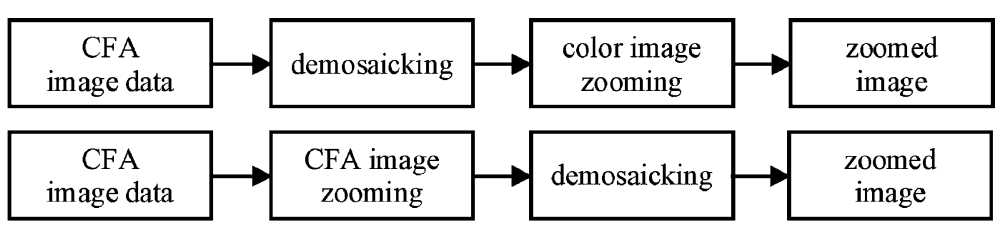

Fig. 4. Image zooming in digital still cameras: (a) demosaicked image zooming operating in the RGB color domain and (b) CFA image operating in the Bayer CFA domain.

zooming technique operating on the RGB color vectors [1], [28], [38];

- CFA image zooming [Fig. 4(b)], which operates directly on the CFA data [12]-[14]. An enlarged CFA image is generated by zooming in the CFA domain. In the sequence, the enlarged CFA image is processed by the demosaicking algorithm producing a full color output image of the increased spatial resolution.

\section{A. Demosaicked Image Zooming}

Most of the demosaicked (color) image zooming methods developed in the last few years are classified into [29]:

- nonadaptive (conventional) interpolation techniques: they utilize an interpolation function, which is indiscriminately applied to the whole images;

- adaptive methods: they analyze the local structure of the source image and then utilize different areas of support to assist the interpolation process.

In general, conventional interpolation techniques such as pixel replication, bilinear interpolation, and spline-based method produce enlarged color images which suffer from aliasing, edge burring, jagged lines or blockiness, and false colors [28]. The degradation in image quality is due to the deviation from the assumptions of ideal low-pass filtering, which forms the basic framework for most of those methods. Other approaches such as those based on Markov random field models produce better results in terms of image fidelity, however, at a higher computational cost due to the large number of design parameters [34]. Adaptive interpolation methods [29], [35]-[37] incorporate structural information into the interpolation process and preserve the image details better when compared to conventional schemes. Finally, it should be noted that all of the above-mentioned methods separately process each color channel, which often leads to the introduction of color artifacts. To alleviate the problem, vector techniques [1], [28], [38] based on the theory of robust order-statistics [39], have been introduced. The vectorial nature of these techniques reduces the presence of most color artifacts and eliminates shifted color edges [1].

Visual inspection of the single-sensor image processing pipeline shown in Fig. 4(a) suggests that the quality of the demosaicked, enlarged output image depends on several factors, not the least of which is the demosaicking scheme's performance. The demosaicking efficiency and the color image zooming precision usually determine the overall performance. It has been widely observed that most demosaicking approaches introduce an initial inaccuracy mainly in the form of blurred edges and false colors [8], [31], [40]. Increasing the spatial resolution of such a demosaicked image, using digital spatial interpolation, means that missing image pixels in the enlarged image are often interpolated from inaccurate estimated values. Therefore, spatial interpolators, both nonadaptive and adaptive, simply amplify imperfections and visual impairments which have been introduced in the preceding demosaicking step. Moreover, digital spatial interpolation in the RGB domain may require the use of an additional software components. The CFA image data zooming [Fig. 4(b)] may represent a more viable alternative in producing enlarged demosaicked images especially for the slim, easy-to-use, high-quality ultrapocket devices and the next generation of digital cameras [12], [13], and its principles are briefly discussed in the next subsection.

\section{B. CFA Image Zooming}

By operating on the CFA data directly [12]-[14], the designers avoid zooming on the RGB vectors where imperfections or noise introduced during demosaicking may create visual impairments. The adaptive technique of [12] and [30] depend on two global parameters which are used to determine the direction in which the interpolation process is directed. The technique operates in a four-stage processing mode in order to complete the enlargement. Since the scheme does not proportionally represent the contribution of the inputs, the method often fails in high-frequency image areas blurring the image. The linear nonadaptive CFA zooming scheme of [14] is a fully automated solution, as it requires no user assistance and does not depend on global parameters. The interpolator in [14] does not utilize any edge information, and it simply performs an averaging operation over the four neighboring inputs. The use of the Bayer pattern configuration and the utilization of the spectral characteristics of the Bayer data significantly boosts its efficiency, making it a candidate solution for CFA image zooming. However, the lack of edge-sensing ability results in blurred fine details and shifted color edges in the final restored (color) output.

Improving the visual quality, especially in terms of image sharpness, can be obtained by using edge-sensing CFA zooming schemes [13], [41]-[43]. The use of edge-sensing weighting coefficients ensures that the zoomer is able to follow the nonlinearities and structural content of the input image. Unfortunately, the utility of the approach in [41] is limited due to the fact that the edge-sensing process is controlled by a user set parameter. Early attempts to introduce an automated, adaptive edge-sensing scheme using weights defined through inversely proportional gradients [42] or aggregated gradients [13], [43] have generated promising results and prompted the development of the framework reported here.

Using the novel and unique processing approach depicted in Fig. 5, the above-listed developments are unified into a new, 


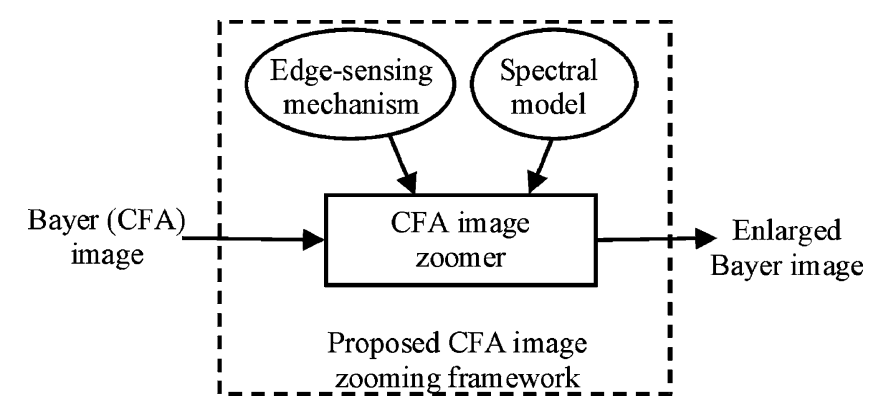

Fig. 5. Unique construction elements used in the proposed CFA image zooming framework.

powerful CFA image zooming framework. The framework employs an edge-sensing mechanism and a spectral model to reduce edge blurring and to minimize the color artifacts present in the enlarged output. By utilizing the twice averaging operator (low-pass filter) in the interpolator's kernel, the proposed approach has been robustified against noise present in the input data. It should be emphasized that the noise-free term is used throughout the paper to denote our assumption that the original (unprocessed) CFA data captured by the sensor are noise-free. This assumption is needed for comparison purposes since all previously defined spatial interpolators operate exclusively on noise-free inputs.

\section{Proposed ZoOMING Scheme}

Let us consider a $K_{1} / k \times K_{2} / k$ Bayer image $z^{\prime}: Z^{2} \rightarrow Z$ with the pixels $z_{(p, q)}$ representing the data received from the A/D converter and $(p, q)$ characterizing the image row (for $p=$ $1,2, \ldots, K_{1} / k$ ) and column (for $q=1,2, \ldots, K_{2} / k$ ). Using the commonly accepted Bayer CFA pattern with the GRGR phase in the first row (Fig. 2), the Bayer image $z^{\prime}$ contains the $\mathrm{R}$ components at (odd $p$, even $q$ ) and the B components at (even $p$, odd $q$ ). The rest of locations, namely (odd $p$, odd $q$ ) and (even $p$, even $q$ ), correspond to the $\mathrm{G}$ components. To increase the spatial resolution of $z^{\prime}$, camera hardware components such as the ASIC and the microprocessor [26] can easily be designed to perform the zooming operation directly on the Bayer data.

Fig. 5 shows the basic construction elements of the proposed framework. As it can be seen, the characteristics of the zooming procedure are essentially determined by the edge-sensing mechanism and the spectral model. The edge-sensing mechanism is used here to preserve the sharpness and structural information of the enlarged image. Its role within the proposed zooming framework will be described in the sequence. The second essential component-spectral model ${ }^{4}$ (Fig. 6) - is used to preserve the spectral correlation that exists between the color components. Since natural RGB images exhibit strong spectral correlation characteristics [1], [15], [16], both researchers and practitioners in the camera image processing community rely on spectral models, such as the color-ratio model [32], the normalized color-ratio models [8], [27], and the color-difference model [33] to eliminate spectral artifacts and color shifts. Following the

\footnotetext{
${ }^{4}$ Any spectral model for CFA image processing is essentially constructed using the spectral quantity formation and spectral normalization blocks [7], [27].
}

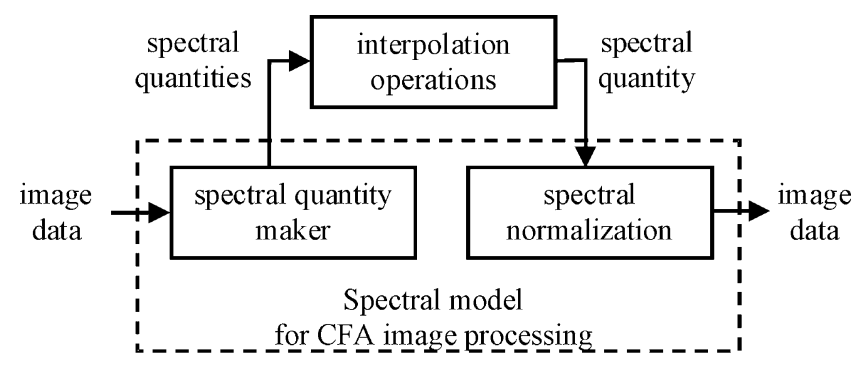

(a)

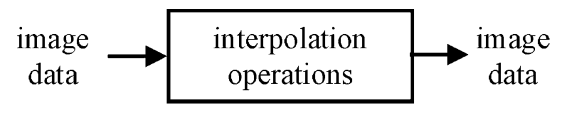

(b)

Fig. 6. Spectral model-based versus conventional interpolator's architectures. (a) The use of the spectral model in the interpolation procedure requires: 1) to transform image data to spectral quantities which are used as the input for an interpolator and 2) to normalize the interpolated, output spectral quantity to obtain the output image intensity. (b) The omission of the spectral model reduces the proposed architecture to the conventional interpolator's architecture operating directly on image data.

dominant contribution of the $\mathrm{G}$ information to human perception and the large number of Bayer G CFA locations, a common feature in all of these models is that they incorporate RG or BG spectral characteristics into the interpolation process. Since the main objective of this study is to introduce the CFA zooming framework, the color-difference model is used throughout this paper as the spectral model, due to its simplicity and pervasive use in the CFA related literature. However, the proposed framework allows easily the utilization of other more sophisticated spectral models such as those described in [8] and [27]. Preliminary results on the use of sophisticated spectral models in demosaicking have been reported recently [7], [27], while an analytical investigation is currently under way.

\section{A. Mapping of the CFA Data Into the Enlarged CFA Image}

Zooming the Bayer CFA data $z^{\prime}$ by a factor of $k$ results in a $K_{1} \times K_{2}$ zoomed Bayer image $z: Z^{2} \rightarrow Z$. The zooming factor $k \in Z$ can be an arbitrary positive integer, however, the value $k=2$ is selected here to facilitate the discussion. Assuming the aforementioned setting, the use of the conventional zooming procedure maps the original CFA data $z_{(p, q)}^{\prime}$ with spatial coordinates $p$ and $q$ into the enlarged image $z$ as follows:

$$
z_{(2 p-1,2 q-1)}=z_{(p, q)}^{\prime}
$$

where the values $z_{(2 p, 2 q)}$ denote the new rows and columns (e.g., of zeros) added to the original data.

Operating on the Bayer data in this way destroys the structure of the Bayer pattern, since R and B components from the original Bayer image $z^{\prime}$ are used to fill in positions reserved for $\mathrm{G}$ samples in the enlarged Bayer image $z$ [Fig. 7(a)]. This atypical arrangement destroys the Bayer mosaic in $z$ and prohibits the subsequent utilization of any Bayer CFA-based demosaicking procedure. In conclusion, conventional zooming approaches cannot be used to perform image enlargement in the Bayer CFA domain. 


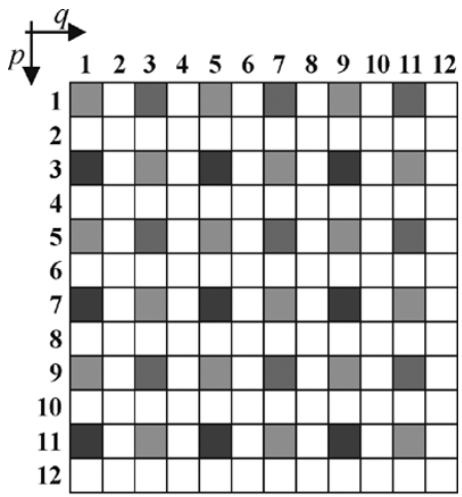

(a)

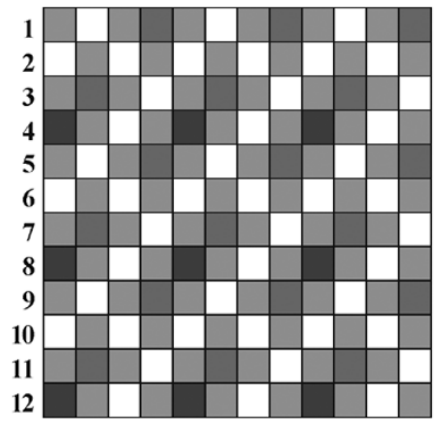

(e)

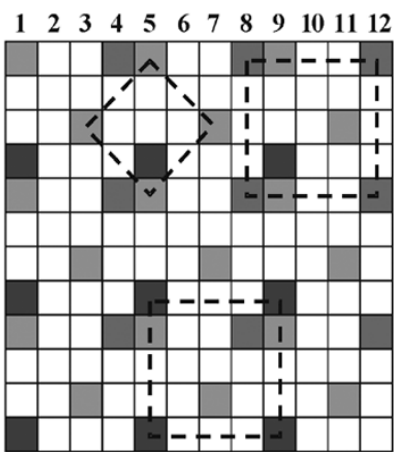

(b)

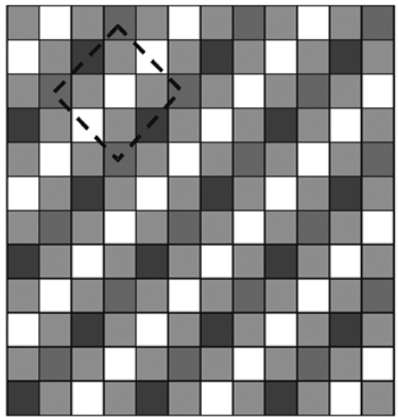

(f)

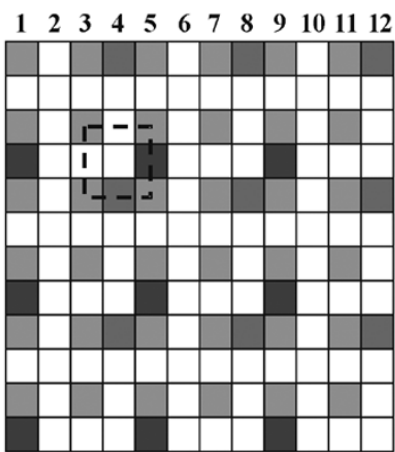

(c)

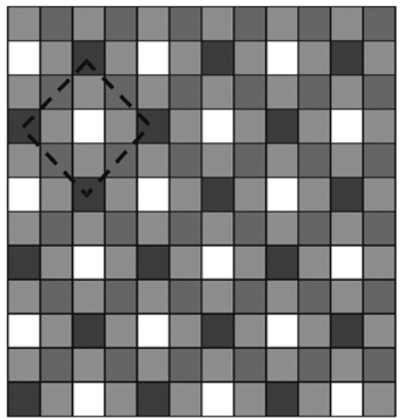

(g)

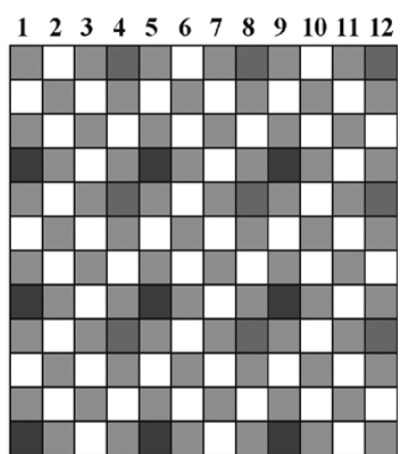

(d)

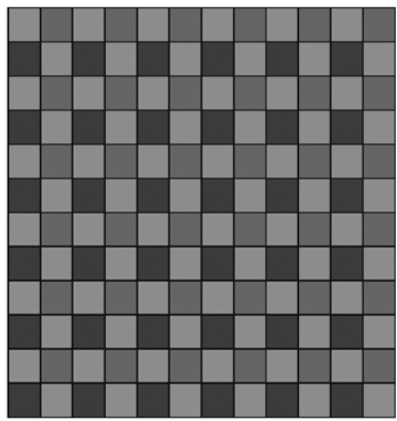

(h)

Fig. 7. Bayer pattern zooming by (a) the standard zooming approach, which destroys the structure of the Bayer pattern (color elements do not correspond to Bayer color positions) and (b) the proposed method continuing in (c)-(h).

To zoom the Bayer data and at the same time preserve the basic structuring assumption behind the CFA, at first the original sensor data should be assigned unique positions corresponding to the proper Bayer structure of the enlarged image as follows [Fig. 7(b)] [13], [14]:

$$
\left.\begin{array}{l}
z_{(2 p-1,2 q)} \\
z_{(2 p, 2 q-1)} \\
z_{(2 p-1,2 q-1)}
\end{array}\right\}=z_{(p, q)}^{\prime}, \quad \begin{aligned}
& \text { for } p \text { odd and } q \text { even } \\
& \text { for } p \text { even and } q \text { odd } \\
& \text { otherwise }
\end{aligned}
$$

where $p$ and $q$ denote coordinates in the original (small) Bayer image $z^{\prime}$.

\section{B. Proposed Edge-Sensing Interpolator for CFA Zooming}

Upon completion of (2), the zooming process interpolates the missing CFA data corresponding to the empty positions of $z$ shown in Fig. 7(b). Due to the fact that the G components occur twice as frequently compared to the $\mathrm{R}$ and the $\mathrm{B}$ values, the proposed algorithm starts the interpolation at pattern locations with missing $\mathrm{G}$ components.

It can be easily observed in Fig. 8(a) that the missing G components are surrounded by four original $\mathrm{G}$ components which form a diamond-shape mask on the image lattice. To proportionally represent the contribution of the adjacent $\mathrm{G}$ components, a missing $\mathrm{G}$ component is obtained using the spatial interpolator shown in Fig. 9(a) as follows:

$$
z_{(p, q)}=\frac{\sum_{\Omega} w_{\left(p_{1}, q_{1}\right)}^{\left(p_{2}, q_{2}\right)} z_{\left(p_{1}, q_{1}\right)}^{\left(p_{2}, q_{2}\right)}}{\sum_{\Omega} w_{\left(p_{1}, q_{1}\right)}^{\left(p_{2}, q_{2}\right)}}
$$

where $\Omega=\left\{\left(p_{1}, q_{1}\right),\left(p_{2}, q_{2}\right) \in \zeta ;\left(p_{1}, q_{1}\right) \neq\left(p_{2}, q_{2}\right)\right\}$. The term $(p, q)$, for $p=1,5, \ldots, K_{1}-3, q=3,7, \ldots, K_{2}-1$ and $p=3,7, \ldots, K_{1}-1, q=1,5, \ldots, K_{2}-3$, denotes the spatial position of the estimated $\mathrm{G}$ values in the enlarged Bayer image $z$ and $\zeta=\{(p-2, q),(p, q-2),(p, q+2),(p+2, q)\}$ denotes the area of support. The averaged values $z_{\left(p_{1}, q_{1}\right)}^{\left(p_{2}, q_{2}\right)}$ are defined using any two of the original $\mathrm{G}$ neighbors $z_{(p-2, q)}, z_{(p+2, q)}, z_{(p, q-2)}$ and $z_{(p, q+2)}$ as follows:

$$
z_{\left(p_{1}, q_{1}\right)}^{\left(p_{2}, q_{2}\right)}=\frac{\left(z_{\left(p_{1}, q_{1}\right)}+z_{\left(p_{2}, q_{2}\right)}\right)}{2}
$$

resulting thus, for $\left(p_{1}, q_{1}\right) \neq\left(p_{2}, q_{2}\right)$, in six possible values $z_{\left(p_{1}, q_{1}\right)}^{\left(p_{2}, q_{2}\right)}$

Visual inspection of Fig. 8(a) reveals that the averaged quantities $z_{(p-2, q)}^{(p+2, q)}$ and $z_{(p, q-2)}^{(p, q+2)}$ are defined in the vertical and horizontal directions, respectively, whereas $z_{(p-2, q)}^{(p, q-2)}, z_{(p-2, q)}^{(p, q+2)}$, $z_{(p+2, q)}^{(p, q-2)}, z_{(p+2, q)}^{(p, q+2)}$ correspond to diagonal directions. Each of the six averaged quantities $z_{\left(p_{1}, q_{1}\right)}^{\left(p_{2}, q_{2}\right)}$ is associated with a positive, real-valued, edge-sensing weighting coefficient $w_{\left(p_{1}, q_{1}\right)}^{\left(p_{2}, q_{2}\right)}$ defined, in general, as follows:

$$
w_{\left(p_{1}, q_{1}\right)}^{\left(p_{2}, q_{2}\right)}=\left[1+f\left(\left|z_{\left(p_{1}, q_{1}\right)}-z_{\left(p_{2}, q_{2}\right)}\right|\right)\right]^{-1}
$$

where $f(\cdot)$ is a function of the absolute difference between the CFA inputs $z_{\left(p_{1}, q_{1}\right)}$ and $z_{\left(p_{2}, q_{2}\right)}$. The properties of a weight such as the one in (5), which is calculated over the inversely proportional gradients $\left|z_{\left(p_{1}, q_{1}\right)}-z_{\left(p_{2}, q_{2}\right)}\right|$ as well as the physical meaning of the definition are discussed in detail in [13] and [27]. 


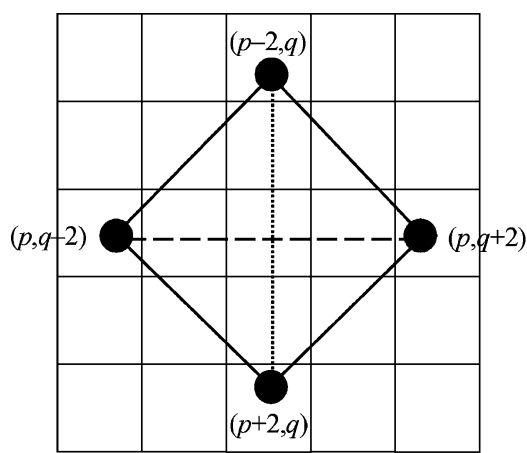

(a)

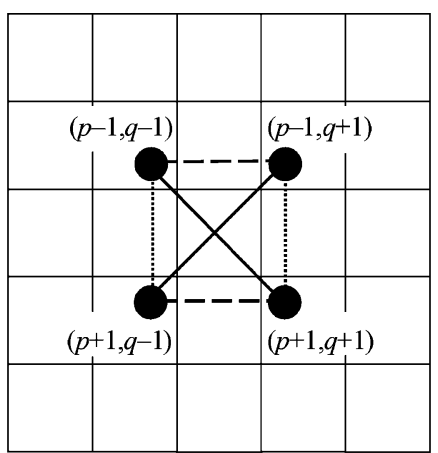

(b)

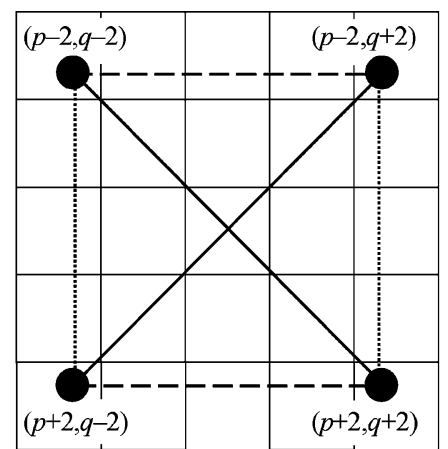

(c)

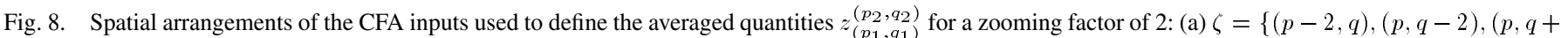

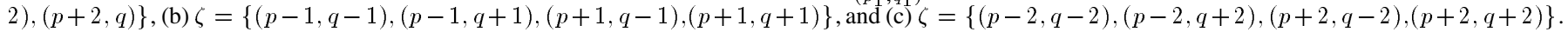

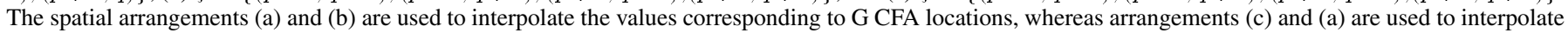

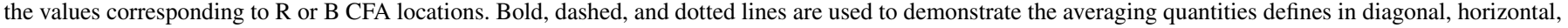
and vertical directions, respectively.

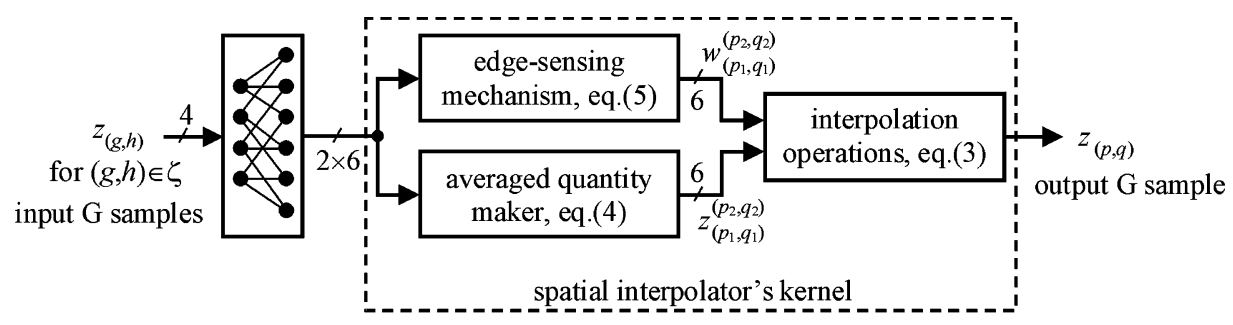

(a)

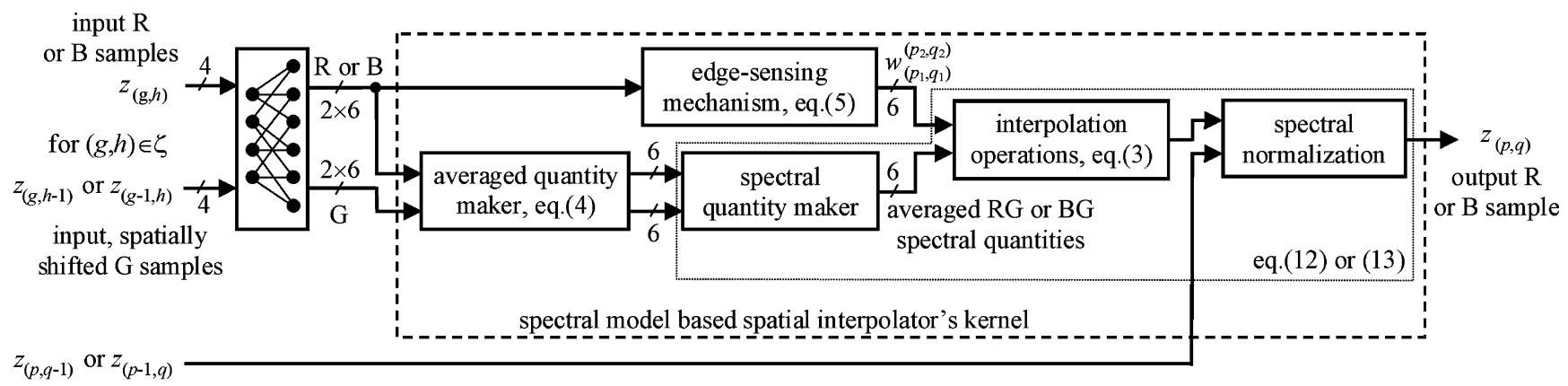

input, spatially shifted

normalizing $\mathrm{G}$ sample

(b)

Fig. 9. Architecture of the proposed zooming solution: (a) spatial interpolator operating over the G samples within the neighborhood $\zeta$ and (b) spectral model-based spatial interpolator operating over R (or B) samples within the neighborhood $\zeta$ and spatially shifted G samples. The framework allows for the utilization of the various edge sensing mechanisms defined in (6)-(11) and/or the various spectral models in [8], [27], [32], [33].

In addition to the properties, the actual shape of the function $f\left(\left|z_{\left(p_{1}, q_{1}\right)}-z_{\left(p_{2}, q_{2}\right)}\right|\right)$ determines the efficiency of the weights' implementation, [44]. A cost-effective, easy-to-implement function is the one initially proposed in [42]

$$
w_{\left(p_{1}, q_{1}\right)}^{\left(p_{2}, q_{2}\right)}=\left[1+\left|z_{\left(p_{1}, q_{1}\right)}-z_{\left(p_{2}, q_{2}\right)}\right|\right]^{-1} .
$$

A more sophisticated form of (6) can be obtained using the aggregated distance concept of [13], [43]

$$
w_{\left(p_{1}, q_{1}\right)}^{\left(p_{2}, q_{2}\right)}=\left[1+\sum_{\Omega}\left(z_{\left(p_{1}, q_{1}\right)}^{\left(p_{2}, q_{2}\right)}-z_{\left(g_{1}, h_{1}\right)}^{\left(g_{2}, h_{2}\right)}\right)\right]^{-1}
$$

where $\Omega=\left\{\left(g_{1}, h_{1}\right),\left(g_{2}, h_{2}\right) \in \zeta ;\left(g_{1}, h_{1}\right) \neq\left(g_{2}, h_{2}\right)\right\}$. However, any improvement in terms of performance is obtained at the expense of higher complexity.

Depending on the design's performance/complexity tradeoff, more computationally advanced solutions can be devised. One such solution uses the concept of the $\alpha$-trimmed mean [39]. Based on the comparisons between the gradient values and the threshold, the extreme observations in the interpolator's input are eliminated. In this way, (3) is robustified against atypical, noise-like, inputs which may degrade the performance if they are left unchecked. The corresponding $\alpha$-trimmed edge-sensing weights are defined as

$$
w_{\left(p_{1}, q_{1}\right)}^{\left(p_{2}, q_{2}\right)}= \begin{cases}1, & \text { if }\left|z_{\left(p_{1}, q_{1}\right)}-z_{\left(p_{2}, q_{2}\right)}\right| \leq \xi \\ 0, & \text { otherwise }\end{cases}
$$


where $\xi$ is a predefined threshold value. If the absolute value of the difference between the inputs is bellow the threshold, the maximum weight value of 1 is assigned to the corresponding interpolator's input $z_{\left(p_{1}, q_{1}\right)}^{\left(p_{2}, q_{2}\right)}$. Otherwise, the weight value is zero, and thus the corresponding $z_{\left(p_{1}, q_{1}\right)}^{\left(p_{2}, q_{2}\right)}$ does not contribute in calculating the output $z_{(p, q)}$ in (3).

Contributions from samples defined across an edge can also be eliminated by thresholding the corresponding weighting coefficient. In such a case, the edge-sensing weights are defined as follows:

$$
w_{\left(p_{1}, q_{1}\right)}^{\left(p_{2}, q_{2}\right)}= \begin{cases}w_{\left(p_{1}, q_{1}\right)}^{\left(p_{2}, q_{2}\right)}, & \text { if } w_{\left(p_{1}, q_{1}\right)}^{\left(p_{2}, q_{2}\right)} \geq \xi \\ 0, & \text { otherwise }\end{cases}
$$

where $\xi$ is a threshold value which provides the end-user with the means to regulate edge sensitivity. The elimination of atypical observations through the use of (9) may result in increased accuracy compared to the accuracy afforded by the solutions in [13], [42], or [43].

It is not difficult to see that the popular nearest-neighbor concept used in demosaicking [45] can be derived from the same framework using the maximum weight $w_{\max } \in$ $\left\{w_{\left(p_{1}, q_{1}\right)}^{\left(p_{2}, q_{2}\right)}\right.$; for $\left.\left(p_{1}, q_{1}\right),\left(p_{2}, q_{2}\right) \in \zeta\right\}$. In this case, the framework determines the weighting coefficients using the following rule:

$$
w_{\left(p_{1}, q_{1}\right)}^{\left(p_{2}, q_{2}\right)}= \begin{cases}1, & \text { if } w_{\left(p_{1}, q_{1}\right)}^{\left(p_{2}, q_{2}\right)}=w_{\max } \\ 0, & \text { otherwise }\end{cases}
$$

The rule suggests that the averaged quantity $z_{\left(p_{1}, q_{1}\right)}^{\left(p_{2}, q_{2}\right)}$ which corresponds to the maximum similarity between the interpolator's inputs $z_{\left(p_{1}, q_{1}\right)}$ and $z_{\left(p_{2}, q_{2}\right)}$ is selected as the output of the operation in (3).

Finally, it should be added that another popular choice for the function $f(\cdot)$ in $(5)$ is the exponential function. As empirical evidence suggests that the relationship between perception and distances measured in physical units is exponential in nature [44], weights in (3) can be defined as follows:

$$
w_{\left(p_{1}, q_{1}\right)}^{\left(p_{2}, q_{2}\right)}=\left[1+\exp \left(-\left|z_{\left(p_{1}, q_{1}\right)}-z_{\left(p_{2}, q_{2}\right)}\right|\right)\right]^{-1} .
$$

Similarly to (11), which represents a perception-oriented alternative to (6), the novel variants of (7) and (8) can be easily obtained by replacing the absolute value term with its exponential form.

In conclusion, any of these weights can be used in the edge-sensing mechanism proposed here. The framework is sufficiently flexible to accommodate weighting functions with different properties and implementation requirements. What is important is that, for the first time, an edge-sensing mechanism suitable for a single-sensor camera is devised using a systematic framework which clearly offers options and flexibility.

\section{Proposed Interpolation Steps for CFA Zooming}

Using the spatial interpolation step (3) with the supporting components defined in (4) and (6), the proposed scheme produces the pattern depicted in Fig. 7(c). Since the spatial interpolation step in (3) does not generate all the needed values

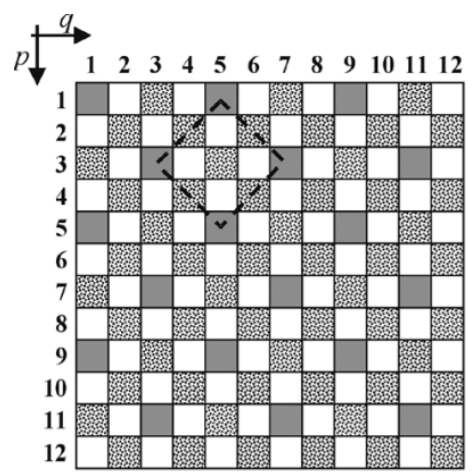

(a)

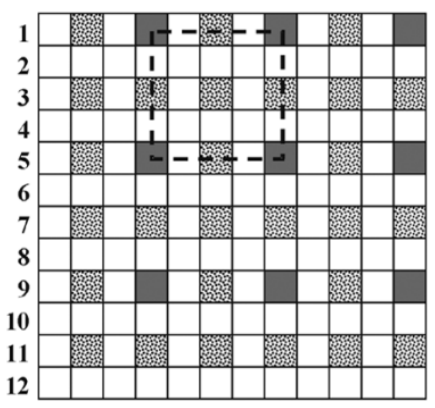

(c)

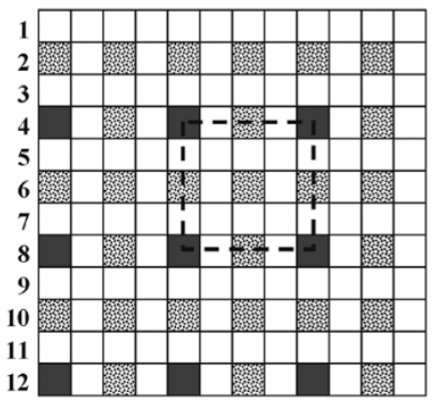

(e)

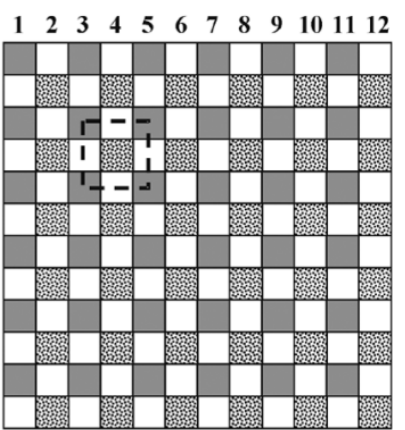

(b)

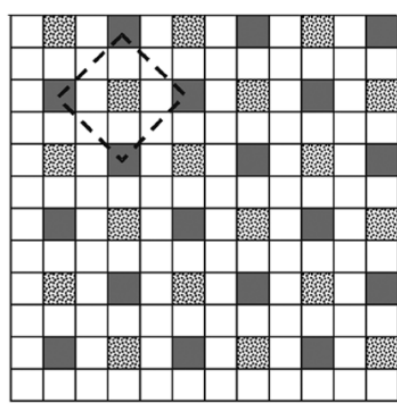

(d)

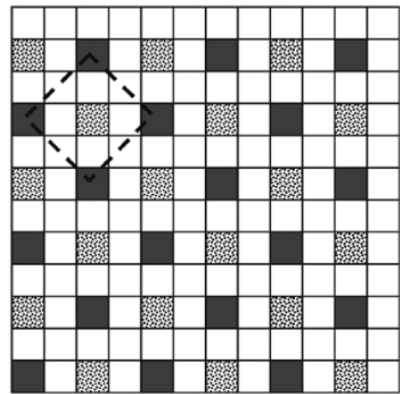

(f)
Fig. 10. Proposed interpolation steps divided into the color planes with the textured missing components. (a) Original $G$ components. (b) G components after the initial $\mathrm{G}$ component interpolation. (c) original $\mathrm{R}$ components. (d) $\mathrm{R}$ components after the initial $\mathrm{R}$ component interpolation. (e) original $\mathrm{B}$ components. (f) B components after the initial B component interpolation.

[Fig. 10(a) and (b)], an additional interpolation step is needed to complete the calculation of the $\mathrm{G}$ components in the enlarged pattern. Fig. 10(a) shows the enlarged Bayer image $z$ with all its original $\mathrm{G}$ components. The locations of the missing $\mathrm{G}$ components correspond to textured pixels. The initial interpolation step (3) is used to interpolate the pixels occupying the center of the four-original $\mathrm{G}$ components which form a diamond-shape mask $\zeta=\{(p-2, q),(p, q-2),(p, q+2),(p+2, q)\}$ on the image lattice. Upon completion of this step, Fig. 10(b) depicts the new situation with the $\mathrm{G}$ components positioned in the center of the square-shape mask described by $\zeta=\{(p-1, q-1),(p-$ $1, q+1),(p+1, q-1),(p+1, q+1)\}$. Therefore, the method continues by interpolating the missing $\mathrm{G}$ components $z_{(p, q)}$, for $p=2,4,6, \ldots, K_{1}-2, q=2,4,6, \ldots, K_{2}-2$, which occupy the center of $\mathrm{G}$ components $z_{(p-1, q-1)}, z_{(p-1, q+1)}, z_{(p+1, q-1)}$ and $z_{(p+1, q+1)}$.

Averaged values $z_{\left(p_{1}, q_{1}\right)}^{\left(p_{2}, q_{2}\right)}$ and the corresponding weighting coefficients $w_{\left(p_{1}, q_{1}\right)}^{\left(p_{2}, q_{2}\right)}$ are calculated using (4) and (6), respectively, 
but now with $\zeta=\{(p-1, q-1),(p-1, q+1),(p+1, q-$ 1), $(p+1, q+1)\}$. As shown in Fig. 8(b), the averaged quantities $z_{(p-1, q-1)}^{(p-1, q+1)}$ and $z_{(p+1, q-1)}^{(p-1, q+1)}$ are defined in diagonal directions, whereas $z_{(p-1, q-1)}^{(p-1, q+1)}, z_{(p+1, q-1)}^{(p+1, q+1)}$ and $z_{(p-1, q-1)}^{(p+1, q-1)}, z_{(p-1, q+1)}^{(p+1, q+1)}$ correspond to horizontal and vertical directions, respectively. The use of (3) with the above-defined support $\zeta$ results in the pattern shown in Fig. 7(d). This concludes the process of determining the $\mathrm{G}$ components.

Analogously to the $\mathrm{G}$ component interpolation, the $\mathrm{R}$ (or B) components are interpolated in two steps [Fig. 10(c)-(f)]. The missing $\mathrm{R}$ components are located at the centers of the squareshape mask [Fig. 10(c)] referenced by the spatial coordinates $p=3,7,11, \ldots, K_{1}-1$ and $q=2,6,10, \ldots, K_{2}-2$. The purpose of this interpolation step is to estimate the $R$ values at the center of a square which has been created by four surrounding original R values $z_{(p-2, q-2)}, z_{(p-2, q+2)}, z_{(p+2, q-2)}$, $z_{(p+2, q+2)}$ occupying the square's corners [Fig. 10(c)]. Since the Bayer image $z^{\prime}$ contains double the number of original $\mathrm{G}$ components compared to original $\mathrm{R}$ or $\mathrm{B}$ components, and the $\mathrm{G}$ channel is considered to be more reliable in terms of original CFA data structural content [3]-[5], both original and interpolated $\mathrm{G}$ components are used to interpolate the $\mathrm{R}$ and $\mathrm{B}$ components [4]-[6], [19]. The proposed spectral model based spatial interpolator, with the structure depicted in Fig. 9(b), uses both spatial and spectral characteristics of the CFA data to produce the output.

Based on the color-difference model of [33], the output of an unbiased, spectral model based spatial interpolator [Fig. 9(b)] can be defined as follows:

$$
z_{(p, q)}=z_{(p, q-1)}+\frac{\sum_{\Omega} w_{\left(p_{1}, q_{1}\right)}^{\left(p_{2}, q_{2}\right)}\left(z_{\left(p_{1}, q_{1}\right)}^{\left(p_{2}, q_{2}\right)}-z_{\left(p_{1}, q_{1}-1\right)}^{\left(p_{2}, q_{2}-1\right)}\right)}{\sum_{\Omega} w_{\left(p_{1}, q_{1}\right)}^{\left(p_{2}, q_{2}\right)}}
$$

where $\Omega=\left\{\left(p_{1}, q_{1}\right),\left(p_{2}, q_{2}\right) \in \zeta ;\left(p_{1}, q_{1}\right) \neq\left(p_{2}, q_{2}\right)\right\}$. The values $w_{\left(p_{1}, q_{1}\right)}^{\left(p_{2}\right)}$ are the weighting coefficients obtained in (6) with the area of support $\zeta=\{(p-2, q-2),(p-2, q+2),(p+$ $2, q-2),(p+2, q+2)\}$. The sample $z_{(p, q-1)}$ in (12) denotes the original $\mathrm{G}$ value neighboring the estimated $\mathrm{R}$ component $z_{(p, q)}$ and $z_{\left(p_{1}, q_{1}\right)}^{\left(p_{2}, q_{2}\right)}-z_{\left(p_{1}, q_{1}-1\right)}^{\left(p_{2},-1\right)}$ are the color-difference quantities defined using R components $z_{\left(p_{1}, q_{1}\right)}, z_{\left(p_{2}, q_{2}\right)}$ and $\mathrm{G}$ components $z_{\left(p_{1}, q_{1}-1\right)}, z_{\left(p_{2}, q_{2}-1\right)}$.

It can be observed that the missing $\mathrm{R}$ component $z_{(p, q)}$ of (12) is neighboring with the G component $z_{(p, q-1)}$. Similarly, the original R components $z_{(p-2, q-2)}, z_{(p-2, q+2)}, z_{(p+2, q-2)}$ and $z_{(p+2, q+2)}$ located at the corners of the square shown in Fig. 10(c) neighbor the original $\mathrm{G}$ components $z_{(p-2, q-3)}$, $z_{(p-2, q+1)}, z_{(p+2, q-3)}, z_{(p+2, q+1)}$. The analogy in spatial shifts between $\mathrm{G}$ and $\mathrm{R}$ components necessitates the use of the added G component $z_{(p, q-1)}$ in (12) so that the output of the weighted sum of averaged RG color differences $z_{\left(p_{1}, q_{1}\right)}^{\left(p_{2}, q_{2}\right)}-z_{\left(p_{1}, q_{1}-1\right)}^{\left(p_{2}, q_{2}-1\right)}$ is normalized in the intensity range [43].

It is not difficult to see that the use of (12) with $p=3,7,11, \ldots, K_{1}-1$ and $q=2,6,10, \ldots, K_{2}-2$ produces the pattern shown in Fig. 7(e). Due to the spatial diagonal symmetry between the original $\mathrm{R}$ and $\mathrm{B}$ positions as

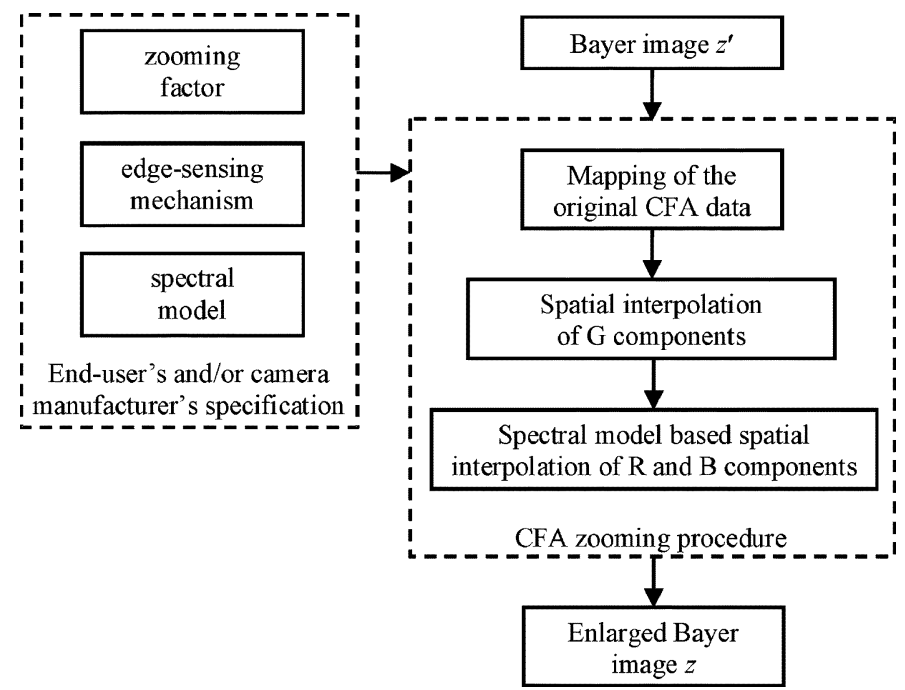

Fig. 11. Block diagram representation of the proposed CFA image zooming framework.

defined in (2), the square-shape masks can be easily reconstituted [Fig. 10(e)] for the purpose of B component interpolation. The missing B components are obtained by modifying the spectral model based spatial interpolator (12) as follows [Fig. 9(b)]:

$$
z_{(p, q)}=z_{(p-1, q)}+\frac{\sum_{\Omega} w_{\left(p_{1}, q_{1}\right)}^{\left(p_{2}, q_{2}\right)}\left(z_{\left(p_{1}, q_{1}\right)}^{\left(p_{2}, q_{2}\right)}-z_{\left(p_{1}-1, q_{1}\right)}^{\left(p_{2}-1, q_{2}\right)}\right)}{\sum_{\Omega} w_{\left(p_{1}, q_{1}\right)}^{\left(p_{2}, q_{2}\right)}}
$$

where $\Omega=\left\{\left(p_{1}, q_{1}\right),\left(p_{2}, q_{2}\right) \in \zeta ;\left(p_{1}, q_{1}\right) \neq\left(p_{2}, q_{2}\right)\right\}$, and $p=2,6,10, \ldots, K_{1}-2$ and $q=3,7,11, \ldots, K_{2}-1$ denote the interpolated locations surrounded by the four original B components $z_{(p-2, q-2)}, z_{(p-2, q+2)}, z_{(p+2, q-2)}, z_{(p+2, q+2)}$. These components along with the four, spatially shifted, $\mathrm{G}$ components $z_{(p-3, q-2)}, z_{(p-3, q+2)}, z_{(p+1, q-2)}, z_{(p+1, q+2)}$ are used to define averaged BG color-difference quantities $z_{\left(p_{1}, q_{1}\right)}^{\left(p_{2}, q_{2}\right)}-z_{\left(p_{1}-1, q_{1}\right)}^{\left(p_{2}-1, q_{2}\right)}$, which calls for the utilization of the normalizing $\mathrm{G}$ component $z_{(p-1, q)}$. Employing the interpolation step (13), the process produces the pattern shown in Fig. 7(f).

In this incomplete mosaic, the missing R (or B) components are located in the center of a diamond-shape mask defined by four R (or B) values $z_{(p-2, q)} z_{(p+2, q)}, z_{(p, q-2)}$ and $z_{(p, q+2)}$, as Fig. 10(d) [or Fig. 10(f)] illustrates. Thus, the rest of the $\mathrm{R}$ values are obtained using the interpolation step of (12) with $p=1,5,9 \ldots, K_{1}-3$ and $q=2,6,10, \ldots, K_{2}-2$ resulting in the pattern shown in Fig. 7(g). Analogously, the missing B components are completed using (13) with $p=2,6,10, \ldots, K_{1}-2$ and $q=1,5,9 \ldots, K_{2}-3$. Both these interpolation steps are based on $\zeta=\{(p-2, q),(p, q-2),(p, q+2)(p+2, q)\}$. This process results in the fully enlarged, interpolated Bayer image $z$ depicted in Fig. 7(h).

A block diagram representation of the proposed CFA image zooming framework is given in Fig. 11 while the interpolation steps performed by the proposed CFA zooming method are summarized, in pseudo-code format, in Fig. 12. From this summary and the construction blocks shown in Fig. 5, it can be claimed that by: 1) replacing the simplest form of the 


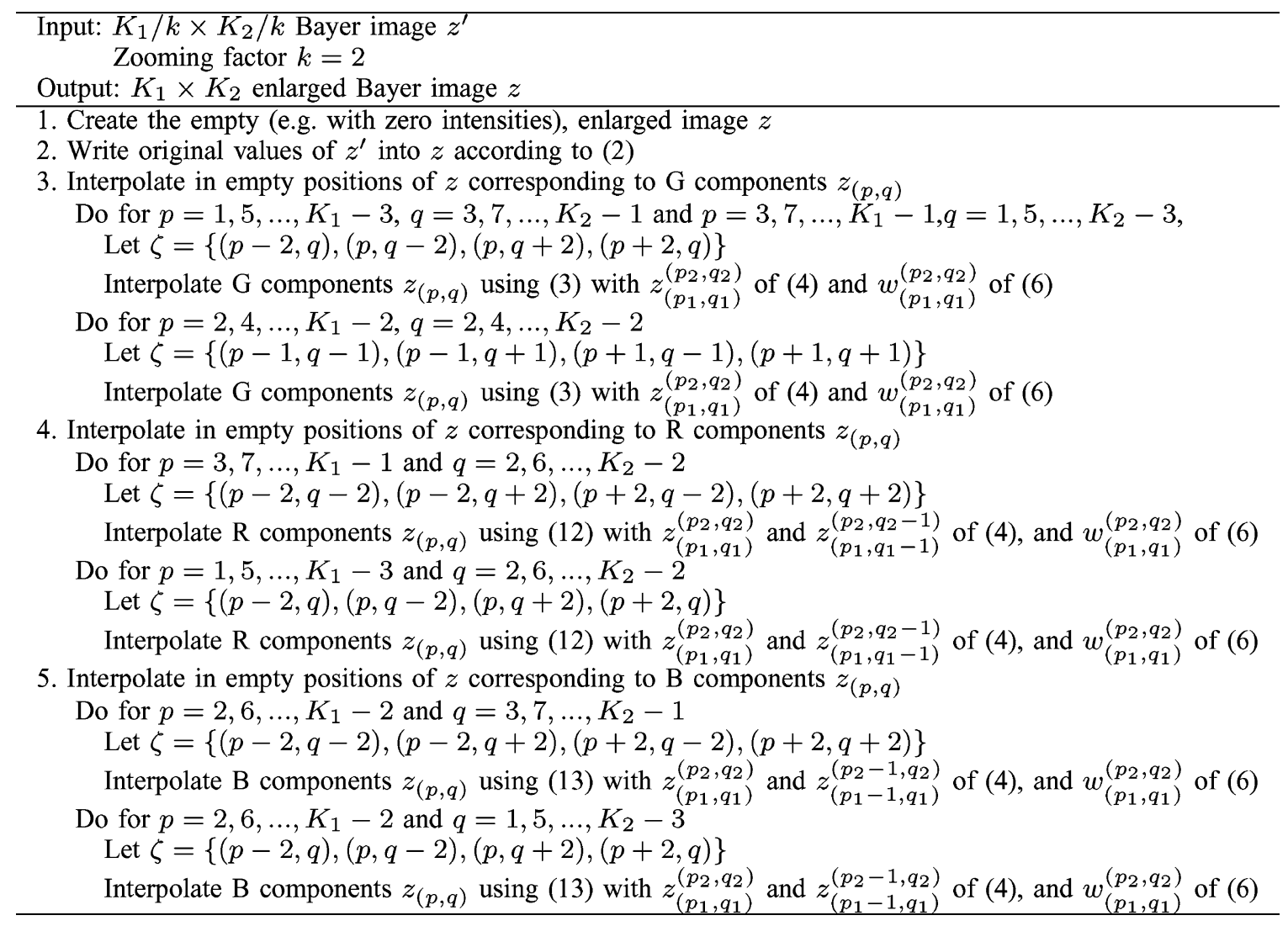

Fig. 12. Pseudocode format of the proposed CFA zooming method.

edge-sensing mechanism (6) employed in (3), (12), and (13) with more sophisticated variants such as those described in previous subsection and/or 2) substituting the color-difference model used in (12) and (13) with the powerful normalized spectral models of [8], [27], the framework can offer solutions which differ in the design philosophy, characteristics, computational complexity, and performance. The enumeration of all available options or the determination of the best configuration of construction elements, according to specified criteria, is beyond the scope of this paper. This study focuses on the development of a zooming framework for CFA data. To demonstrate the suitability of the proposed framework, the simplest solution belonging to this class is devised (Fig. 12) and used in the sequence for comparison purposes. Studies on the determination of the optimal structure and issues related to the unification of the CFA zooming, demosaicking and postprocessing [8] algorithmic steps will be the subject of a forthcoming submission.

\section{EXPERIMENTAL RESULTS}

The proposed zooming method is tested using the color test database ${ }^{5}$ shown in Fig. 13. These images have been captured using three-sensor cameras or color scanners and they have been extensively used by practitioners and researchers working in camera image processing, especially in demosaicking. This is mainly due to the fact that the database: 1) contains natural,

\footnotetext{
${ }^{5}$ The used color image database is posted online at www.dsp.utoronto.ca/ حlukacr/images8.zip.
}

real-life images; 2) represents real-life scenarios, and 3) has images that vary in complexity and color appearance. Note that, in order to facilitate comparisons, all images have been normalized to the standard $512 \times 512,8$-bit per channel RGB representation.

\section{A. Problem Formulation}

To measure the efficiency of the zooming methods objectively, the approach shown in Fig. 14 is used. The process starts with the $K_{1} \times K_{2}$ original color image $\mathbf{o}: Z^{2} \rightarrow Z^{3}$ which is down-sampled with a factor of 2 to produce the $K_{1} / 2 \times K_{2} / 2$ color image $\mathrm{o}^{\prime}: Z^{2} \rightarrow Z^{3}$. Following the procedure of [12], the down-sampling operations are realized here by averaging the original RGB vectors as follows:

$\mathbf{o}_{(p, q)}^{\prime}=\operatorname{mean}\left\{\mathbf{o}_{(2 p-1,2 q-1)}, \mathbf{o}_{(2 p-1,2 q)}, \mathbf{o}_{(2 p, 2 q-1)}, \mathbf{o}_{(2 p, 2 q)}\right\}$

where $(p, q)$ denotes the spatial position in the small, downsampled image $\mathbf{o}^{\prime}$.

Upon completion of the down-sampling step, the image $\mathbf{o}^{\prime}$ is then transformed into the $K_{1} / 2 \times K_{2} / 2$ Bayer image $z^{\prime}: Z^{2} \rightarrow$ $Z$ as follows [4], [7]:

$$
z_{(p, q)}^{\prime}= \begin{cases}o_{(p, q) 1}^{\prime}, & \text { for } p \text { odd and } q \text { even } \\ o_{(p, q) 3}^{\prime}, & \text { for } p \text { even and } q \text { odd } \\ o_{(p, q) 2}^{\prime}, & \text { otherwise }\end{cases}
$$

where $\mathbf{o}_{(p, q)}^{\prime}=\left[o_{(p, q) 1}^{\prime}, o_{(p, q) 2}^{\prime}, o_{(p, q) 3}^{\prime}\right]$ denotes the color vector in a $K_{1} / 2 \times K_{2} / 2$ down-sampled image $\mathbf{o}^{\prime}$ with spatial co- 


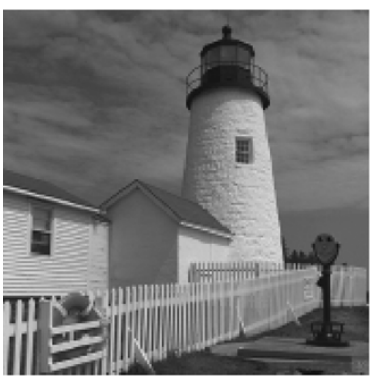

(a)

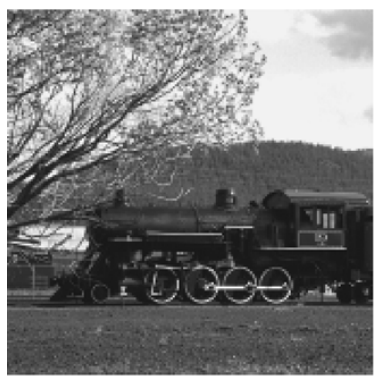

(e)

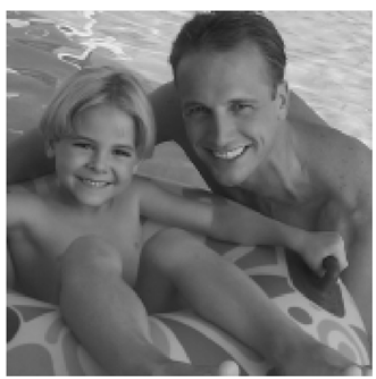

(b)

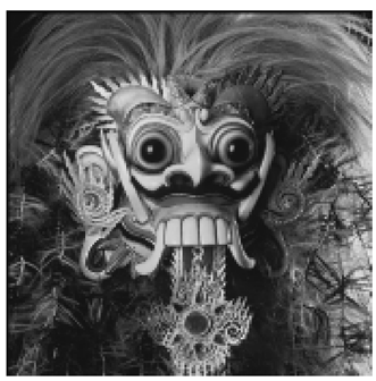

(f)

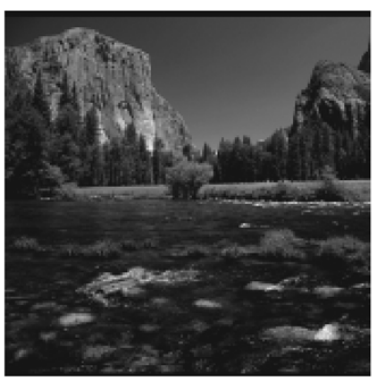

(c)

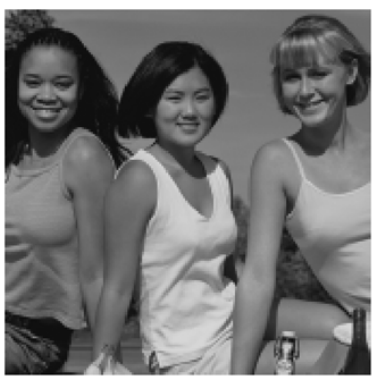

(g)

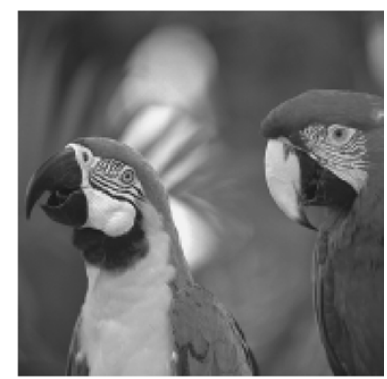

(d)

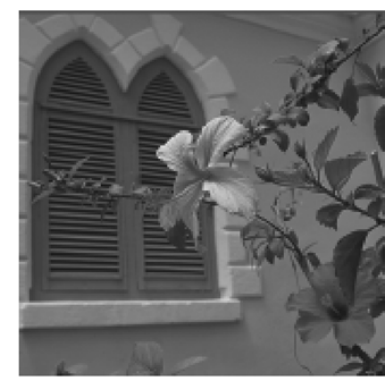

(h)

Fig. 13. Used test image database.

ordinates $p=1,2, \ldots, K_{1} / 2$ and $q=1,2, \ldots, K_{2} / 2$. Since the original Bayer image is usually unavailable, researchers resort to the approach of (15) in order to obtain test Bayer image data used for demosaicking [3], [19], [40], filtering [2], zooming [12], [14], and compression [11] comparative evaluations.

Using the proposed CFA-zooming method, we can obtain the zoomed $K_{1} \times K_{2}$ Bayer image $z: Z^{2} \rightarrow Z$. It is known that half of the pixels $z_{(p, q)}$ correspond to the $\mathrm{G}$ channel, whereas the $\mathrm{R}$ and $\mathrm{B}$ channels are assigned the other half of the pixels in the Bayer pattern. Assuming that $p=1,2, \ldots, K_{1}$ and $q=$ $1,2, \ldots, K_{2}$ denote the spatial position of the pixels in vertical (image rows) and horizontal (image columns) directions shown in Fig. 14, gray-scale pixels $z_{(p, q)}$ can be transformed into the RGB vectors $\mathbf{x}_{(p, q)}=\left[x_{(p, q) 1}, x_{(p, q) 2}, x_{(p, q) 3}\right]$, using the following transformation [4], [7]:

$$
\mathbf{x}_{(p, q)}= \begin{cases}{\left[z_{(p, q)}, 0,0\right],} & \text { for } p \text { odd and } q \text { even } \\ {\left[0,0, z_{(p, q)}\right],} & \text { for } p \text { even and } q \text { odd } \\ {\left[0, z_{(p, q)}, 0\right],} & \text { otherwise. }\end{cases}
$$

This transformation results in a $K_{1} \times K_{2}$ RGB image $\mathbf{x}: Z^{2} \rightarrow$ $Z^{3}$ representing a two-dimensional (2-D) matrix with three-dimensional (3-D) vector samples. Note that each color vector $\mathbf{x}_{(p, q)}$ relates to one true component varying in $k=1,2,3$ of $x_{(p, q) k}$ from position to position, whereas the other two components of $\mathbf{x}_{(p, q)}$ are set to zero.

Using demosaicking methods, the missing color components of $\mathbf{x}$ are obtained. The resulting $K_{1} \times K_{2}$ interpolated RGB image $\mathbf{y}: Z^{2} \rightarrow Z^{3}$ should be as close as possible to the desired RGB image $\mathbf{o}$. This design objectively corresponds to the minimization of the expected value of the mean square error as follows:

$$
\text { minimize } E\left\{\|\mathbf{o}-\mathbf{y}\|^{2}\right\}
$$

where $E\{\cdot\}$ indicates statistical expectation guaranteeing the minimum average loss or risk.

The difference between the original $\mathbf{o}$ and the interpolated image $\mathbf{y}$ constitutes the error image $\mathbf{e}=\|\mathbf{o}-\mathbf{y}\|: Z^{2} \rightarrow Z^{3}$. This error is the result of both the zoomer's inaccuracy and the limitations of the demosaicking method.

To objectively compare the efficiency of the proposed zooming method, we will make use of two approaches, namely, the so-called demosaicked image zooming approach shown in Fig. 4(a), and the proposed CFA image zooming approach shown in Fig. 4(b). It is evident that the efficiency of both approaches depends on the demosaicking. To this end, we will use the most commonly utilized Bayer CFA-based demosaicking solutions, such as the alternating projection (AP) approach [3], the correlation-correction approach (CCA) [4], the adaptive color plane interpolation (API) scheme [18], the bilinear difference (BD) interpolation scheme [19], and the bilinear interpolation (BI) scheme [31], [45].

Following the conventional camera zooming paradigm of Fig. 4(a), first the selected demosaicking algorithm is applied to the original Bayer data $z^{\prime}$ of (15). The obtained color image output $\mathbf{y}^{\prime}$ is then enlarged by applying standard image zooming algorithms, such as the (bi)linear zooming (BICZ) scheme [46] and bicubic zooming (BCCZ) scheme [47], [48]. The spatially enlarged color image $\mathbf{y}$ is considered to be the output of the overall process.

For the novel camera zooming approach [Fig. 4(b)], the proposed CFA zooming algorithm as well as the locally adaptive CFA zooming algorithm (LZ) [12] and the advanced linear averaging CFA zooming (AAZ) scheme [14] are applied to the original Bayer data $z^{\prime}$. The enlarged CFA image $z$ is produced at the end of this step. In the sequence, the selected demosaicking scheme is applied to generate the enlarged color image $\mathbf{y}$, which forms the output of the overall process. 

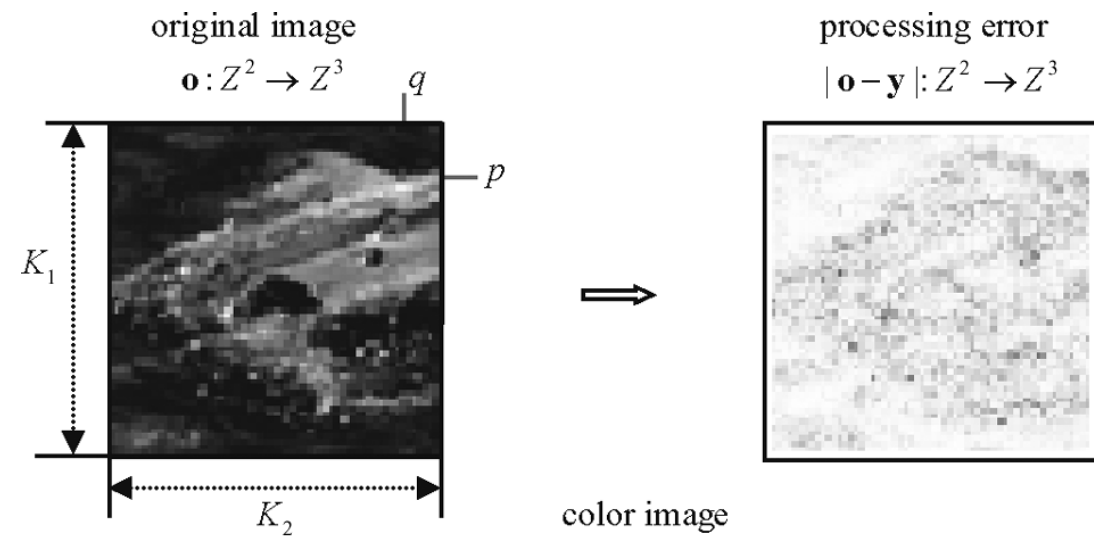

restored (demosaicked) image

color image

$\mathbf{x}^{\prime}: Z^{2} \rightarrow Z^{3}$

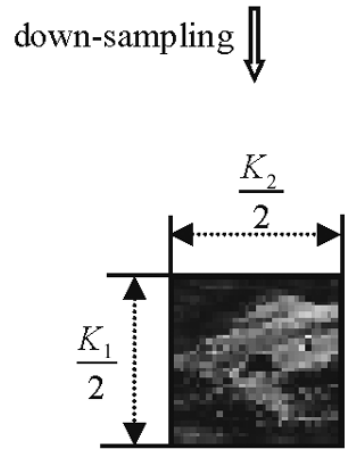

down-sampled image

$\mathbf{o}^{\prime}: Z^{2} \rightarrow Z$
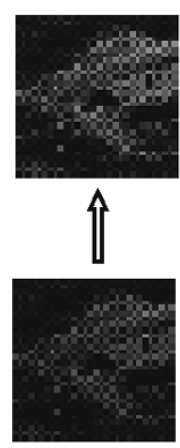

Bayer image

$z^{\prime}: Z^{2} \rightarrow Z$

Fig. 14. Procedure used for objective evaluation of results.

\section{B. Performance Evaluation}

To measure the similarity between the original o and interpolated image $\mathbf{y}$ with the spatial dimensions of $K_{1} \times K_{2}$ pixels, a number of different objective measures based on the difference in statistical distributions of the pixel values can be utilized. In this paper, the mean absolute error (MAE), the mean square error (MSE), and the normalized color-difference (NCD) criteria [16] are considered.

The MAE and MSE criteria are defined as follows:

$$
\begin{aligned}
\mathrm{MAE} & =\frac{1}{3 K_{1} K_{2}} \sum_{k=1}^{3} \sum_{p=1}^{K_{1}} \sum_{q=1}^{K_{2}}\left|o_{(p, q) k}-x_{(p, q) k}\right| \\
\mathrm{MSE} & =\frac{1}{3 K_{1} K_{2}} \sum_{k=1}^{3} \sum_{p=1}^{K_{1}} \sum_{q=1}^{K_{2}}\left(o_{(p, q) k}-x_{(p, q) k}\right)^{2}
\end{aligned}
$$

where $\mathbf{o}_{(p, q)}=\left[o_{(p, q) 1}, o_{(p, q) 2}, o_{(p, q) 3}\right]$ is the original pixel and $\mathbf{x}_{(p, q)}=\left[x_{(p, q) 1}, x_{(p, q) 2}, x_{(p, q) 3}\right]$ is the restored pixel, with $(p, q)$ denoting a spatial position in a $K_{1} \times K_{2}$ color image and $k$ characterizing the color channel.

To quantify the perceptual similarity between the original and the obtained solution, the NCD criterion is used as follows:

$$
\mathrm{NCD}=\frac{\sum_{p=1}^{z K_{1}} \sum_{q=1}^{z K_{2}} \sqrt{\sum_{k=1}^{3}\left(\bar{o}_{(p, q) k}-\bar{y}_{(p, q) k}\right)^{2}}}{\sum_{p=1}^{z K_{1}} \sum_{q=1}^{z K_{2}} \sqrt{\sum_{k=1}^{3}\left(\bar{o}_{(p, q) k}\right)^{2}}}
$$

TABLE I

COMPARISON OF THE STANDARD COLOR IMAGE ZOOMERS USING THE AvERAGED RESUlts CORRESPONDING TO THE DATABASE IN FIG. 13

\begin{tabular}{c|ccc|ccc}
\hline Color zooming & \multicolumn{3}{|c|}{ BICZ } & \multicolumn{3}{c}{ BCCZ } \\
\hline CFA int. / Crit. & MAE & MSE & NCD & MAE & $M S E$ & $N C D$ \\
\hline BI & 9.90 & 358.9 & 0.0998 & 9.75 & 354.6 & 0.1039 \\
BD & 8.71 & 265.9 & 0.0840 & 8.58 & 258.7 & 0.0849 \\
AP & 8.69 & 257.6 & 0.0810 & 8.58 & 250.2 & 0.0816 \\
API & 8.60 & 268.6 & 0.0820 & 8.48 & 262.9 & 0.0833 \\
CCA & 8.39 & 251.3 & 0.0736 & 8.24 & 242.7 & 0.0735 \\
\hline
\end{tabular}

where $\overline{\mathbf{o}}_{(p, q)}=\left[\bar{o}_{(p, q) 1}, \bar{o}_{(p, q) 2}, \bar{o}_{(p, q) 3}\right]$ and $\overline{\mathbf{y}}_{(p, q)}=$ $\left[\bar{y}_{(p, q) 1}, \bar{y}_{(p, q) 2}, \bar{y}_{(p, q) 3}\right]$ are the vectors representing the RGB vectors $\mathbf{o}$ and $\mathbf{y}$, respectively, in the CIE LUV color space [49].

\section{Achieved Results}

Results presented in Tables I and II correspond to zooming and restoration of the test images shown in Fig. 13. These results demonstrate the robustness of the proposed method, since the error values are calculated as aggregated measures averaged through the number of images in the test database (Fig. 13). Note that Table I summarizes results achieved using the conventional zooming schemes, which zoom the (demosaicked) RGB color images obtained after demosaicking. Table II summarizes results obtained by applying the CFA zooming scheme followed by the demosaicking scheme in cascade. Comparing these results, it can be observed that the reported error values depend critically on the demosaicking efficiency. Therefore, the CFA zooming techniques followed by the CCA demosaicking 
TABLE II

COMPARISON OF THE CFA IMAge ZOOMERs Using the AVERAGED RESUlts CoRRESPONDING TO THE DATABASE IN FIG. 13

\begin{tabular}{c|ccc|ccc|ccc}
\hline Bayer zooming & \multicolumn{3}{|c|}{ LZ } & \multicolumn{3}{c|}{ AAZ } & \multicolumn{3}{c}{ Proposed zooming } \\
\hline CFA int. / Crit. & $M A E$ & $M S E$ & $N C D$ & $M A E$ & $M S E$ & $N C D$ & $M A E$ & $M S E$ & $N C D$ \\
\hline BI & 10.89 & 425.29 & 0.1151 & 9.29 & 304.4 & 0.1073 & 9.00 & 298.65 & 0.1048 \\
BD & 10.56 & 390.79 & 0.1045 & 8.95 & 284.7 & 0.0974 & 8.68 & 275.83 & 0.0944 \\
AP & 10.82 & 401.14 & 0.1012 & 9.11 & 289.4 & 0.0971 & 8.87 & 282.96 & 0.0938 \\
API & 10.63 & 399.33 & 0.1079 & 9.08 & 287.0 & 0.1021 & 8.79 & 283.89 & 0.0983 \\
CCA & 10.49 & 386.18 & 0.0969 & 8.92 & 276.9 & 0.0921 & 8.61 & 270.74 & 0.0868 \\
\hline
\end{tabular}

scheme or the CCA demosaicking scheme followed by conventional color image zooming in the RGB domain produce the best results. Other combinations of demosaicking schemes (AP, API, BD, BI) and zooming algorithms produce results inferior to those reported by the CCA demosaicking scheme. It should be also emphasized that the cost-effective pocket devices, mobile phones, and imaging devices for surveillance and automotive applications are restricted in their computational resources and, thus, expensive demosaicking solutions such as AP, CCA, or API can hardly be embedded in these devices. Therefore, the objective of these comparisons is to find the zooming solution producing the highest image quality while employing the cost-effective BI demosaicking scheme. The results indicate that, in this set of processing cascades, the proposed zooming framework significantly outperforms other solutions in terms of both MAE and MSE values. Moreover, excellent design characteristics of the proposed CFA zooming framework always result in the best values of objective criteria among the CFA zooming methods and the significant improvement compared to previously developed LZ and AAZ CFA zooming schemes in terms of all objective measures.

Fig. 15 allows the visual comparison of the original images, down-sampled images, and the enlarged outputs achieved using the $\mathrm{BI}$ demosaicking scheme combined with the BCCZ zooming and the proposed CFA zooming approach followed by the CCA demosaicking scheme. These results illustrate that the standard approach (BI demosaicking + BCCZ zooming) blurs image edges, structural content, and fine details. Moreover, the corresponding outputs depicted in Fig. 15(c) contain a number of color artifacts comparing with the original images shown in Fig. 15(a). However, the proposed CFA zooming method followed by the CCA demosaicking scheme preserves the image details and eliminates color artifacts. This results in naturally colored outputs such as those depicted in Fig. 15(d).

Figs. 16 and 17 present zoomed parts of the restored images and allow the comparison of many methods, in terms of the subjective evaluation of the restored images. It is evident that inaccurate zooming performance [Fig. 16(b) and (c) and Fig. 17(b) and (c)] significantly affects the restored image quality, even when the powerful demosaicking schemes (i.e., BD, API, and CCA) are applied. Inappropriate zooming leads to strong color artifacts and significantly blurred image edges. Since many interpolation methods fail near edges [Fig. 16(b)-(d) and Fig. 17(b)-(d)] and produce color artifacts [3], [4], [40], design of an efficient zooming algorithm is a key to performance. Following the structural information of the Bayer data, the proposed zooming scheme is capable of enlarging the color images with a high visual quality and avoids many image imperfections related to previously intro- duced zooming methods. Morevover, the proposed framework provides the coloration which is much truer to the original. Combining with the efficient demosaicking solution such as the CCA scheme, the proposed CFA zooming method results in impressive visual quality of the restored images shown in Fig. 16(1) and Fig. 17(1).

Since the down-sampling operations, such as those in (14), may affect the overall performance in the evaluation approach of Fig. 14, an evaluation test using the original images without down-sampling is performed. Each one of the $K_{1} \times K_{2}$ original images is sampled with the Bayer CFA to obtain the "equivalent" sensor data, and then the enlarged $2 K_{1} \times 2 K_{2}$ color image is generated by applying either the demosaicked image zooming [Fig. 4(a)] or the CFA image zooming [Fig. 4(b)] processing pipelines. The image quality is evaluated subjectively, only due to the lack of an original input with the appropriate spatial dimensions. Visual inspection of the results depicted in Fig. 18(a) and (b) and Fig. 19(a) and (b) suggests that the conventional zooming solution generates the images which suffers from zipper effects, color shifts, and blurred edges. The amount of visual impairments is reduced, if not eliminated, when the proposed framework is applied. The results presented in Fig. 18(c) and (d) and Fig. 19(c) and (d) clearly indicate that the proposed CFA zooming framework produces the highest visual quality also in this case.

Summarizing the results presented above, the following conclusions can be drawn.

- Employing a cost-effective demosaicking solutions such as the BI scheme, the proposed CFA zooming method outperforms both the previously introduced CFA-based zooming schemes and conventional image zooming methods operating on demosaicked (RGB color) image outputs, both in terms of objective and subjective evaluation methods.

- The proposed framework utilizes the additional information afforded by the employed spectral model along with the structure of the Bayer pattern to accurately estimate missing values of the enlarged CFA image. In addition, the employed edge-sensing mechanism allows us to fully utilize the structural content of the Bayer data. By utilizing the readily available knowledge regarding the underlying structure, the proposed zooming scheme achieves significant improvements in terms of objective and subjective evaluation of the image quality.

- The proposed method can zoom at an arbitrary zooming factor, preserving every time the underlying structure of the Bayer color data array.

- The proposed method curbs the proliferation of imperfections introduced during the demosaicking process. The 
(a)
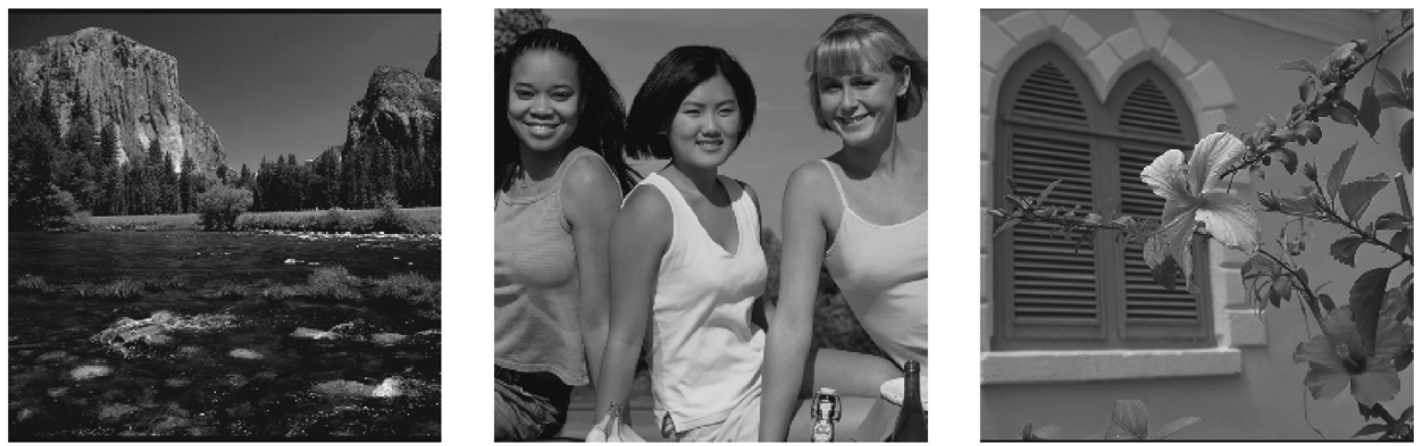

(b)

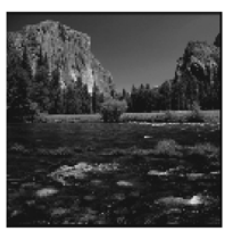

(c)
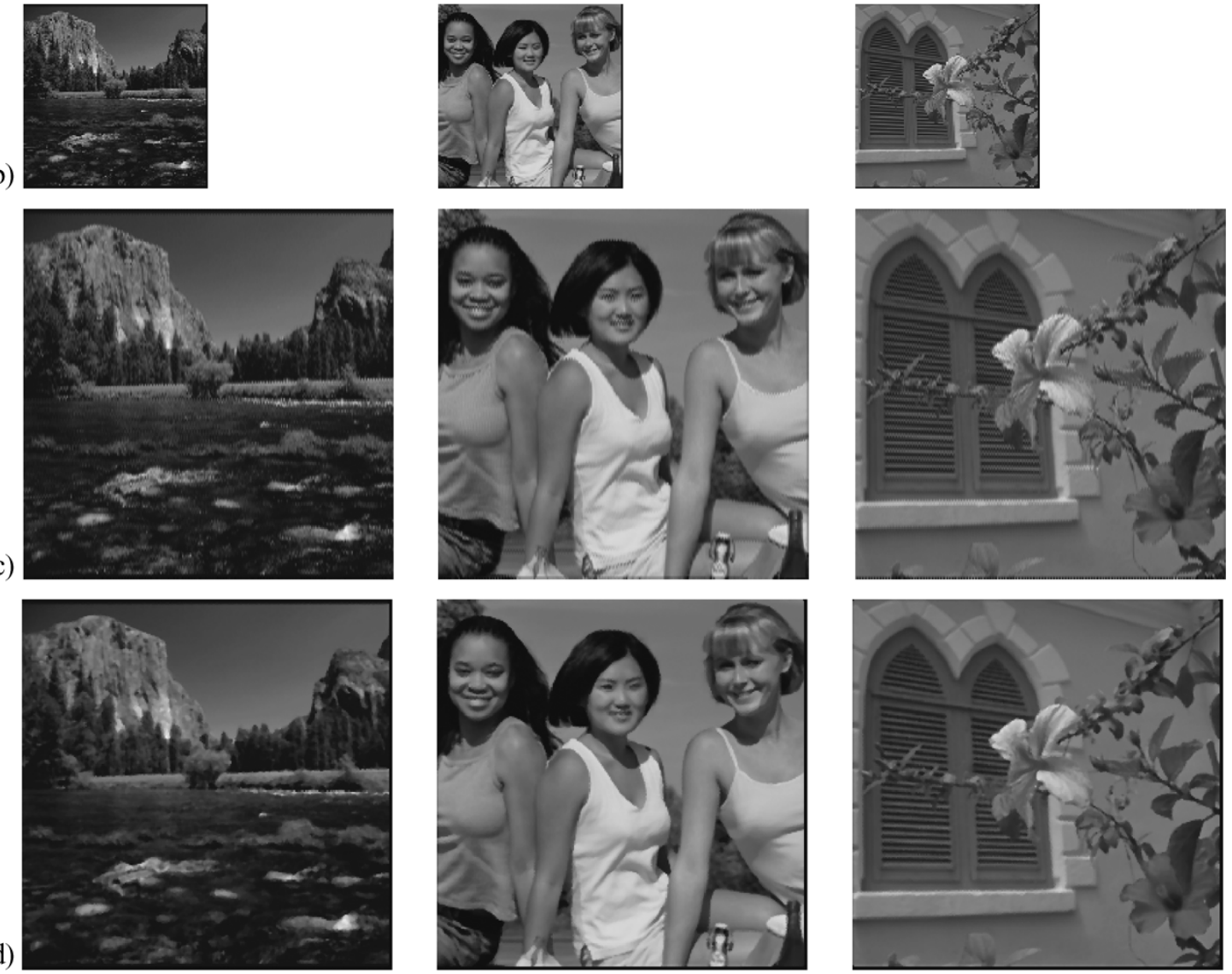

Fig. 15. Visual extremes comparing the images corresponding to Fig. 13(c), (g), and (h). (a) Original images. (b) Down-sampled images. (c) BI demosaicking + BCCZ zooming. (d) Proposed CFA zooming + CCA demosaicking.

edge-sensing mechanism and spectral model used in our adaptive CFA zooming framework helps us to produce sharp, visually pleasing, zoomed color images.

\section{COMPUtational COMPLEXity}

Apart from the numerical behavior (actual performance) of the proposed algorithm, its computational complexity is a realistic measure of its practicality and usefulness. Therefore, the proposed method is analyzed here in terms of normalized operations, such as additions (ADDs), subtractions (SUBs), divisions (DIVs), multiplications (MULTs) and absolute values (ABSVs).

The spatial interpolator (3) depicted in Fig. 9(a) is used to interpolate the missing $\mathrm{G}$ components. Each one of the six averaged quantities $z_{\left(p_{1}, q_{1}\right)}^{\left(p_{2}, q_{2}\right)}$ of (3) requires one ADD and one DIV for its evaluation. Each of the corresponding weighting coefficients requires to realize one DIV, ADD, ABSV, and SUB. Counting together six MULTs, ten ADDs, and one DIV represent the cost of implementing the normalized weighting operation in (3). Thus, the total number of operations needed to recover the missing $\mathrm{G}$ component per interpolated location is

$O_{\mathrm{G}}=22 \mathrm{ADDs}+6 \mathrm{SUBs}+13 \mathrm{DIVs}+6 \mathrm{MULTs}+6 \mathrm{ABSVs}$.

When the spectral model based spatial interpolator (12) or (13) is used to estimate the R or B components [Fig. 9(b)], respectively, two additional SUBs per color-difference quantity evaluation are necessary. Moreover, one extra ADD, corresponding to the use of the normalizing $\mathrm{G}$ component $z_{(p, q-1)}$ in 


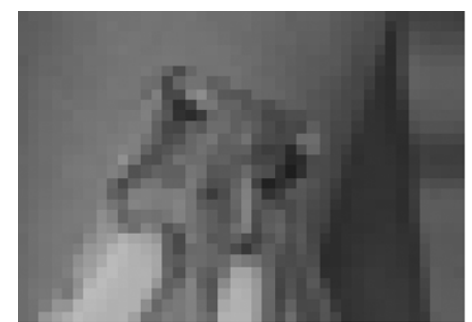

(a)

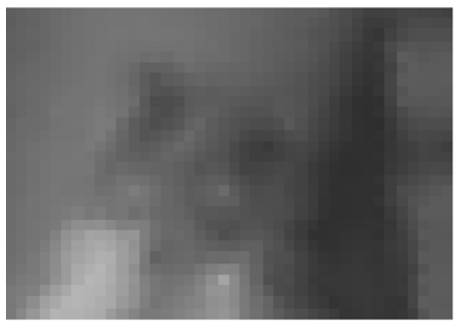

(d)

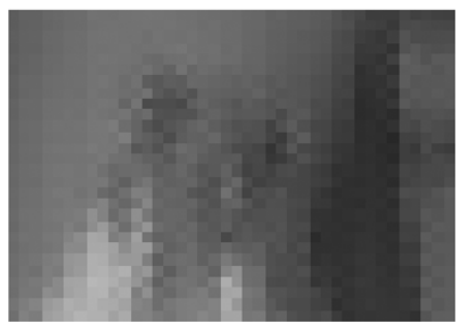

(g)

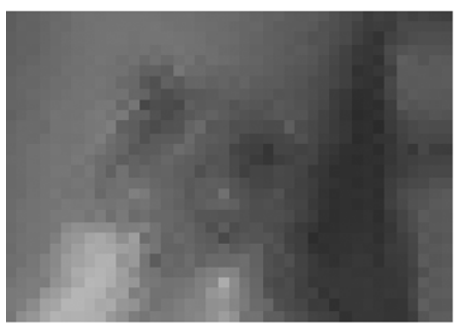

(j)

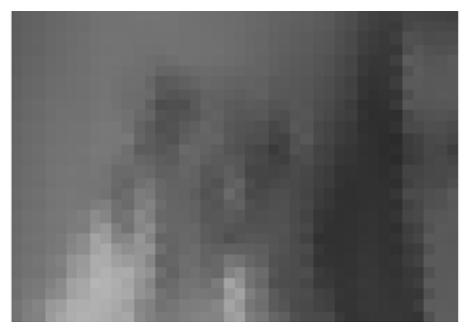

(b)

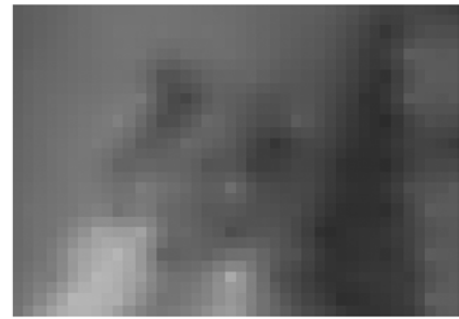

(e)

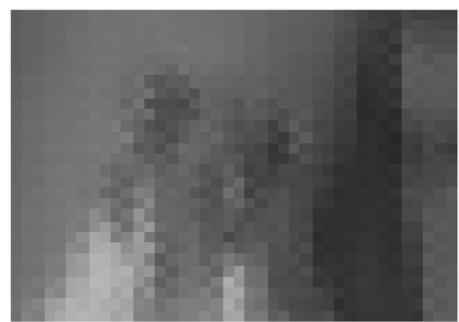

(h)

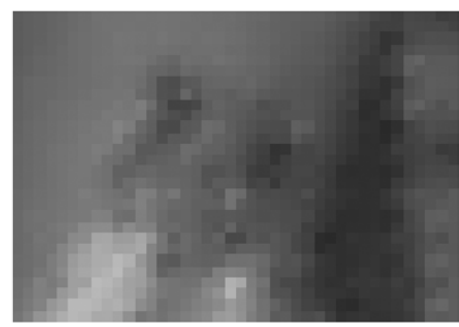

(k)

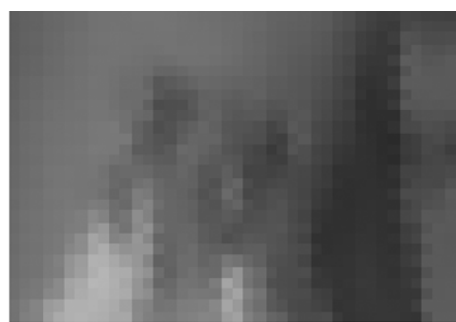

(c)

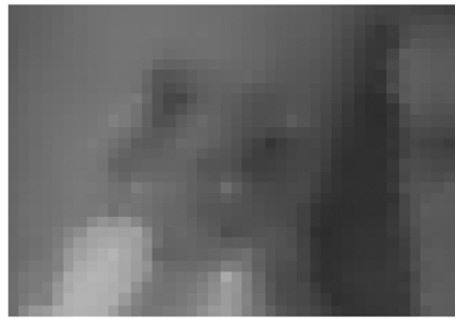

(f)

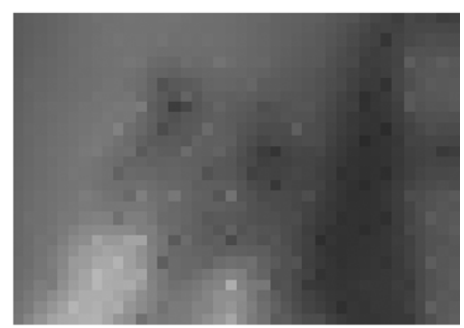

(i)

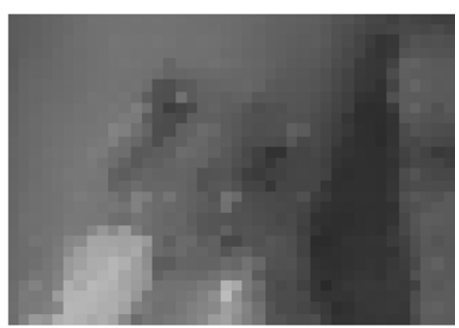

(1)

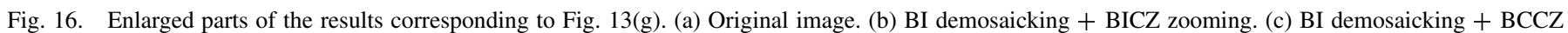

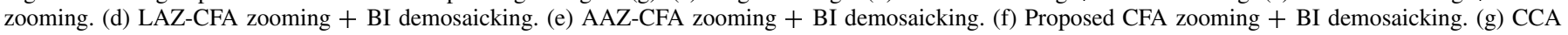

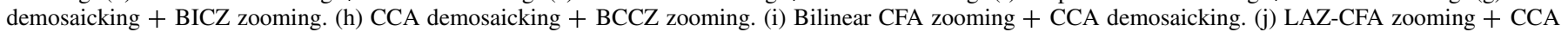
demosaicking. (k) AAZ-CFA zooming + CCA demosaicking. (l) Proposed CFA zooming + CCA demosaicking.

(12) or $z_{(p-1, q)}$ in (13), should be added. Thus, the total number of operations per $\mathrm{R}$ or $\mathrm{B}$ component location is

$$
O_{\mathrm{RB}}=O_{\mathrm{G}}+1 \mathrm{ADDs}+12 \mathrm{SUBs} .
$$

Since the color-difference model of (12) is not used during RGB-based zooming, implementing the proposed method on the color vector domain decreases the number of spectral subtractions. However, RGB-based zooming requires operations to be performed on each color channel. Thus, the total cost associated with the RGB based implementation per spatial location is

$$
O_{\mathrm{RGB}}=3 O_{\mathrm{G}} \text {. }
$$

It can be therefore argued that, by performing zooming in the CFA domain, significant computational savings can be obtained. The relative advantage increases with both the image size and the zooming factor. The proof of this claim can be easily done by counting the total cost per a whole image.

Let us consider a zooming factor $k$ and a $K_{1}^{\prime} \times K_{2}^{\prime}$ (small) Bayer image $z^{\prime}$. The zooming scheme output is always of size $\left(k K_{1}^{\prime} \times k K_{2}^{\prime}\right)$ pixels. This suggests that $\left[\left(k^{2}-1\right) K_{1}^{\prime} K_{2}^{\prime}\right]$ samples need to be interpolated. The $\mathrm{G}$ components represent half of $\left[\left(k^{2}-1\right) K_{1}^{\prime} K_{2}^{\prime}\right]$ missing components in the Bayer image $z$. Therefore, the total number of operations needed during interpolation at the $\mathrm{G}$ locations is $\left[O_{G}\left(k^{2}-1\right) K_{1}^{\prime} K_{2}^{\prime}\right] / 2$, where division by 2 indicates that only half of the interpolated locations refer to the $\mathrm{G}$ components in the enlarged Bayer image $z$. Analogously, the total number of operations necessary during interpolation of 


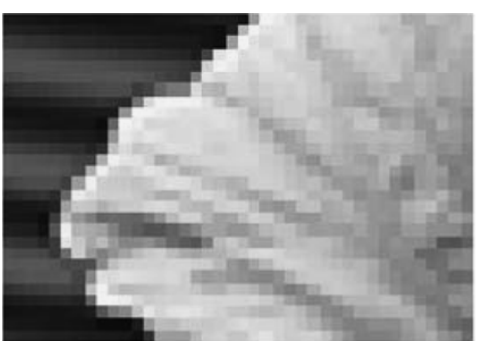

(a)

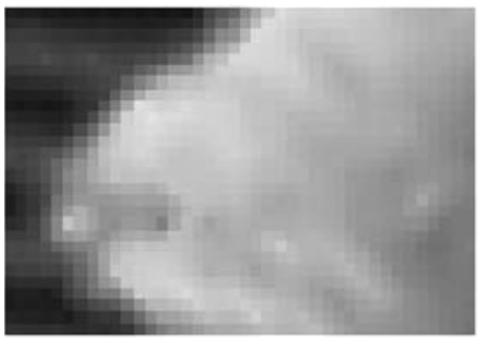

(d)

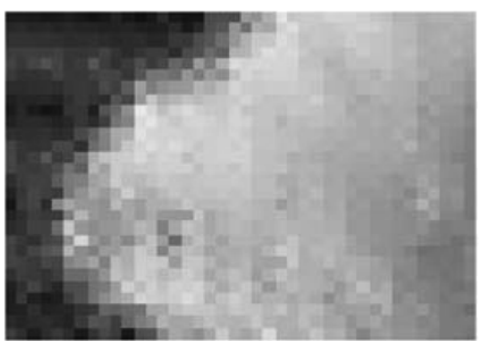

$(\mathrm{g})$

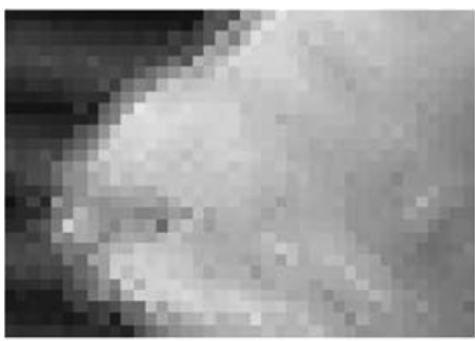

(j)

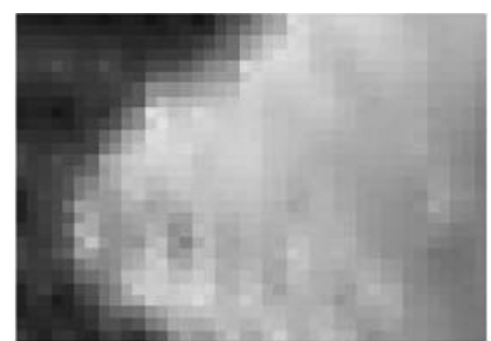

(b)

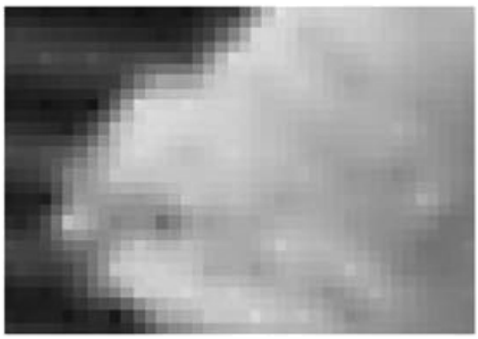

(e)

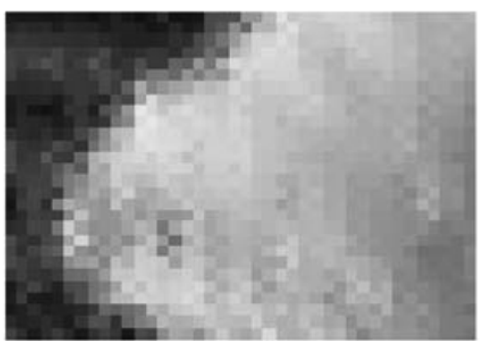

(h)

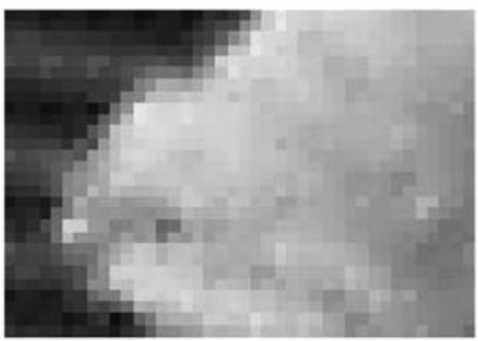

(k)

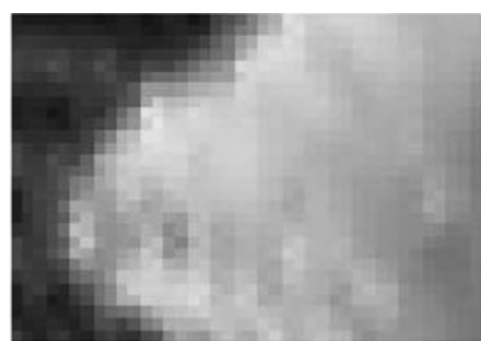

(c)

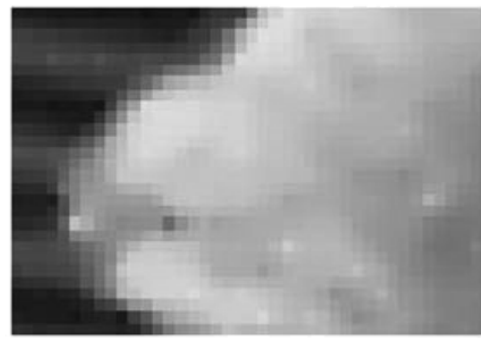

(f)

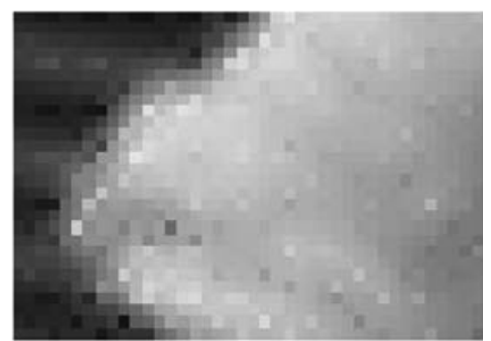

(i)

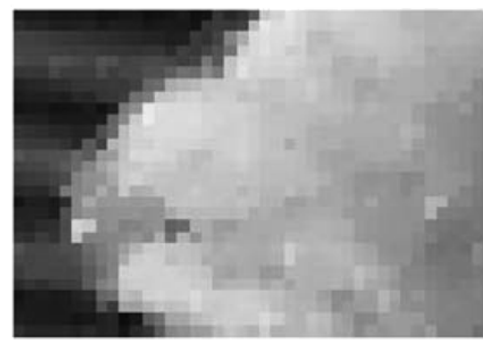

(1)

Fig. 17. Enlarged parts of the results corresponding to Fig. 13(h). (a) Original image. (b) BI demosaicking + BICZ zooming. (c) BI demosaicking + BCCZ zooming. (d) LAZ-CFA zooming + BI demosaicking. (e) AAZ-CFA zooming + BI demosaicking. (f) Proposed CFA zooming + BI demosaicking. (g) CCA demosaicking + BICZ zooming. (h) CCA demosaicking + BCCZ zooming. (i) Bilinear CFA zooming + CCA demosaicking. (j) LAZ-CFA zooming + CCA demosaicking. (k) AAZ-CFA zooming + CCA demosaicking. (1) Proposed CFA zooming + CCA demosaicking.

the $\mathrm{R}$ and $\mathrm{B}$ samples is $\left[O_{R B}\left(k^{2}-1\right) K_{1}^{\prime} K_{2}^{\prime}\right] / 2$. In summary, the proposed CFA zooming method performs

$$
\begin{aligned}
O_{\mathrm{CFA}_{\text {total }}}=\left(k^{2}-1\right) K_{1}^{\prime} K_{2}^{\prime}[22.5 \mathrm{ADDs}+12 \mathrm{SUBs} \\
+13 \mathrm{DIVs}+6 \mathrm{MULTs}+6 \mathrm{ABSVs}]
\end{aligned}
$$

operations during interpolation of the missing values in the enlarged CFA image $z$.

In the case of the RGB-based zooming, the adaptive spatial interpolator (3) requires performing $O_{\mathrm{RGB}}$ at all $\left(k^{2}-1\right) K_{1}^{\prime} K_{2}^{\prime}$ interpolated locations. Thus, the total number of operations per whole enlarged image in the RGB-based implementation is

$O_{\mathrm{RGB}_{\text {total }}}=\left(k^{2}-1\right) K_{1}^{\prime} K_{2}^{\prime}[66 \mathrm{ADDs}+18 \mathrm{SUBs}$

$$
+39 \mathrm{DIVs}+18 \mathrm{MULTs}+18 \mathrm{ABSVs}]
$$

which represents a significant expense compared to (24). The difference in the cost between these two approaches significantly increases with both the spatial dimensions of the $K_{1}^{\prime} \times K_{2}^{\prime}$ input image and the zooming factor $k$.

The analysis suggests that the proposed zooming framework can be considered a computationally efficient method useful for practical, cost-effective camera solutions. Potentially demanding solutions such as those utilizing the edge-sensing mechanisms defined in (7)-(11) or their other variants may be implemented in a companion personal computer (PC) which interfaces with the digital camera which stores the images in the raw (CFA) format. In this case, a PC-based software application supports the zoomer and, thus, the parameter $\xi$ in 


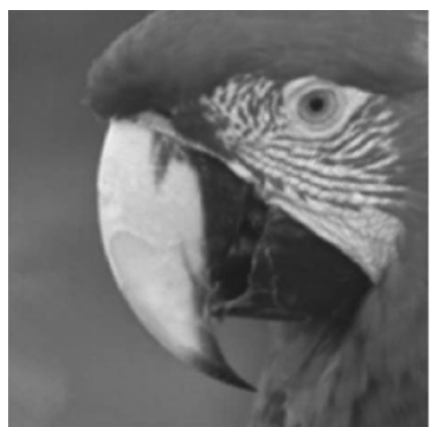

(a)

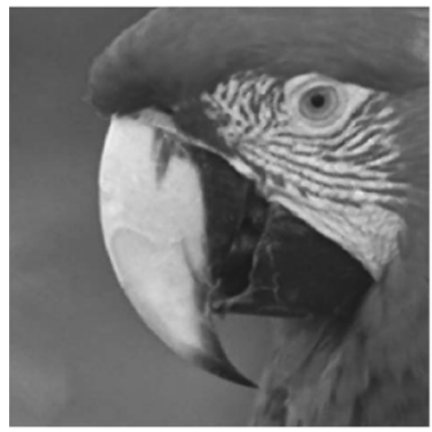

(c)

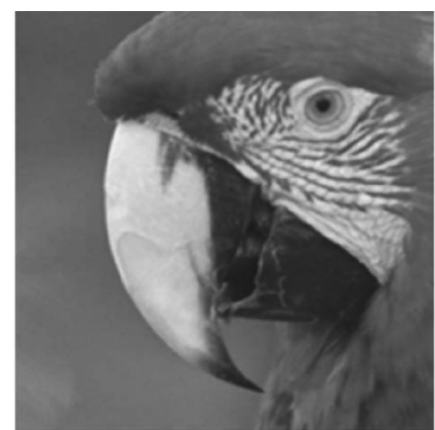

(b)

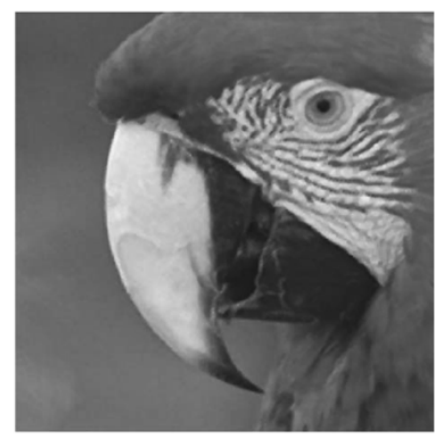

(d)

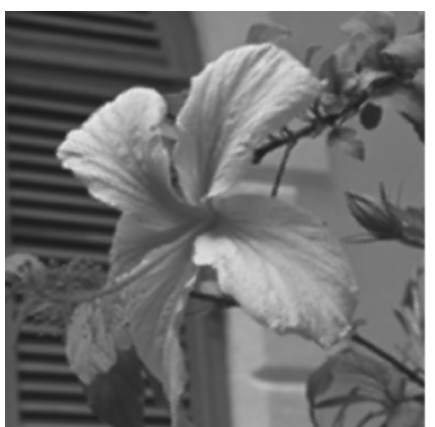

(a)

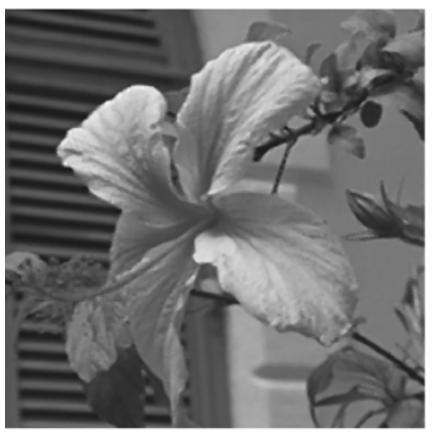

(c)

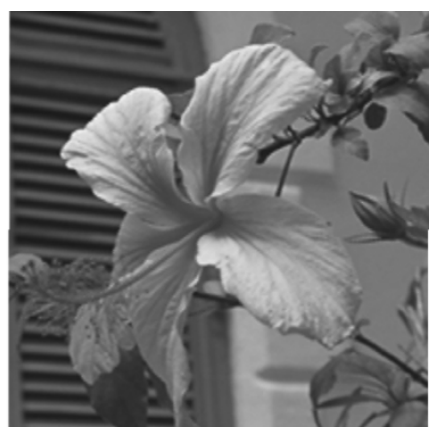

(b)

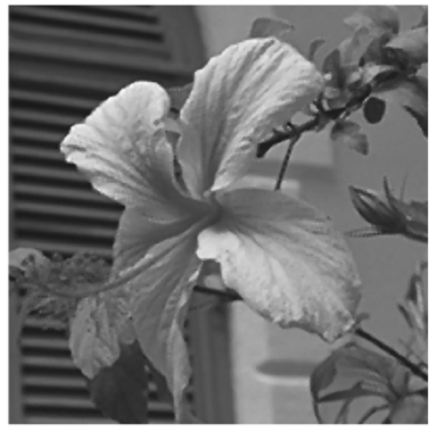

(d)
Fig. 18. Enlarged parts of the results achieved using Fig. 13(d). (a) BI demosaicking + BICZ zooming. (b) CCA demosaicking + BICZ zooming. (c) Proposed CFA zooming + BI demosaicking. (d) proposed CFA zooming + CCA demosaicking.

(8) or (9) can be optimally set, based on subjective evaluations, by the end user.

Finally, the efficiency of the zooming schemes is measured, in terms of the execution time, using a conventional PC equipped with a commonly used operating system and a standard programming environment. When implemented in software, the execution of the proposed zooming tool on a PC with an Intel Pentium IV 2.40-GHz CPU, 512-MB RAM, Windows XP operating system, and MS Visual C++ 5.0 programming environment took (on average) $0.22 \mathrm{~s}$ per a $256 \times 256$ pattern to be enlarged. As opposed to this case, the BICZ, BCCZ, LZ, and AAZ took $0.25,0.48,0.24$, and $0.15 \mathrm{~s}$, respectively. The recorded values suggest that the proposed procedure represents an efficient and cost-effective image zooming solution. It should be noted that the objective of the comparisons performed between the BICZ, BCCZ, LZ, AAZ, and the proposed zooming procedure is to provide benchmark information regarding implementation issues. The development of software-optimized realizations of the algorithms under consideration is beyond the scope of this paper.

\section{CONCLUSION}

A novel zooming framework operating on Bayer CFA data was introduced and analyzed. The framework employed an edge sensing mechanism and a color-difference model. Combining their advantages, our solution enlarges Bayer images, while preserving edges and structural contents. Used in conjunction with the demosaicking procedure, the introduced zooming algorithm
Fig. 19. Enlarged parts of the results achieved using Fig. 13(h). (a) BI demosaicking + BICZ zooming. (b) CCA demosaicking + BICZ zooming. (c) Proposed CFA zooming + BI demosaicking. (d) Proposed CFA zooming + CCA demosaicking.

avoids color artifacts and produces enlarged color images pleasurable for viewing. At the same time it yields excellent results in terms of commonly used objective image quality criteria. Simulation results and comparisons reported here indicate that the proposed framework is sufficiently robust, computationally efficient and relatively easy to implement. Moreover, employing the effective BI demosaicking procedure the proposed cost-effective solution can significantly outperform previously developed approaches which utilize the identical BI demosaicking step.

\section{REFERENCES}

[1] R. Lukac, B. Smolka, K. Martin, K. N. Plataniotis, and A. N. Venetsanopulos, "Vector filtering for color imaging," IEEE Signal Process. Mag., vol. 22, no. 1, pp. 74-86, Jan. 2005.

[2] A. Bosco, K. Findlater, S. Battiato, and A. Castorina, "A noise reduction filter for full-frame data imaging devices," IEEE Trans. Consumer Electron., vol. 49, no. 3, pp. 676-682, Aug. 2003.

[3] B. Gunturk, Y. Altunbasak, and R. Mersereau, "Color plane interpolation using alternating projections," IEEE Trans. Image Process., vol. 11, no. 9, pp. 997-1013, Sep. 2002.

[4] R. Lukac, K. N. Plataniotis, D. Hatzinakos, and M. Aleksic, "A novel cost effective demosaicing approach," IEEE Trans. Consumer Electron., vol. 50, no. 1, pp. 256-261, Feb. 2004.

[5] W. Lu and Y. P. Tang, "Color filter array demosaicking: new method and performance measures," IEEE Trans. Image Process., vol. 12, no. 10, pp. 1194-1210, Oct. 2003.

[6] L. Chang and Y. P. Tang, "Effective use of spatial and spectral correlations for color filter array demosaicking," IEEE Trans. Consumer Electron., vol. 50, no. 1, pp. 355-365, May 2004. 
[7] R. Lukac and K. N. Plataniotis, "Data adaptive filters for demosaicking: a framework," IEEE Trans. Consumer Electron., vol. 51, no. 2, pp. 560-570, May 2005.

[8] R. Lukac, K. Martin, and K. N. Plataniotis, "Demosaicked image postprocessing using local color ratios," IEEE Trans. Circuits Syst. Video Technol., vol. 14, no. 6, pp. 914-920, Jun. 2004.

[9] T. Toi and M. Ohita, "A subband coding technique for image compression in single CCD cameras with Bayer color filter arrays," IEEE Trans. Consumer Electron., vol. 45, no. 1, pp. 176-180, Feb. 1999.

[10] S. Y. Lee and A. Ortega, "A novel approach of image compression in digital cameras with a Bayer color filter array," in Proc. Int. Conf. Image Processing, vol. 3, Oct. 2001, pp. 482-485.

[11] C. C. Koh, J. Mukherjee, and S. K. Mitra, "New efficient methods of image compression in digital cameras with color filter array," IEEE Trans. Consumer Electron., vol. 49, no. 4, pp. 1448-1456, Nov. 2003.

[12] S. Battiato, G. Gallo, and F. Stanco, "A locally adaptive zooming algorithm for digital images," Image Vis. Comput., vol. 20, no. 11, pp. 805-812, Sep. 2002.

[13] R. Lukac and K. N. Plataniotis, "Digital camera zooming on the color filter array," Electron. Lett., vol. 39, no. 25, pp. 1806-1807, Dec. 2003.

[14] — - "Digital zooming for color filter array based image sensors," RealTime Imaging, vol. 11, no. 2, pp. 129-138, Apr. 2005.

[15] G. Sharma, Digital Color Imaging Handbook. Boca Raton, FL: CRC Press, 2002.

[16] K. N. Plataniotis and A. N. Venetsanopoulos, Color Image Processing and Applications. Berlin, Germany: Springer-Verlag, 2000.

[17] B. E. Bayer, "Color Imaging Array," U.S. Patent 3971 065, Jul. 20, 1976.

[18] J. F. Hamilton and J. E. Adams, "Adaptive Color Plane Interpolation in Single Sensor Color Electronic Camera," U.S. Patent 5629 734, May 13, 1997

[19] S. C. Pei and I. K. Tam, "Effective color interpolation in CCD color filter arrays using signal correlation," IEEE Trans. Circuits Syst. Video Technol., vol. 13, no. 6, pp. 503-513, Jun. 2003.

[20] H. J. Trussell and R. E. Hartwig, "Mathematics for demosaicking," IEEE Trans. Image Process., vol. 11, no. 4, pp. 485-492, Apr. 2002.

[21] P. L. P. Dillon, D. M. Lewis, and F. G. Kaspar, "Color imaging system using a single CCD area array," IEEE J. Solid-State Circuits, vol. SSC-13, no. 1, pp. 28-33, Feb. 1978

[22] B. T. Turko and G. J. Yates, "Low smear CCD camera for high frame rates," IEEE Trans. Nucl. Sci., vol. 36, no. 1, pp. 165-169, Feb. 1989.

[23] A. J. Blanksby and M. J. Loinaz, "Performance analysis of a color CMOS photogate image sensor," IEEE Trans. Electron Devices, vol. 47, no. 1, pp. 55-64, Jan. 2000.

[24] T. Lule, S. Benthien, H. Keller, F. Mutze, P. Rieve, K. Seibel, M. Sommer, and M. Bohm, "Sensitivity of CMOS based imagers and scaling perspectives," IEEE Trans. Electron Devices, vol. 47, no. 11, pp. 2110-2122, Nov. 2000

[25] D. R. Cok, "Single-Chip Electronic Color Camera With Color-Dependent Birefringent Optical Spatial Frequency Filter and Red and Blue Signal Interpolating Circuit,” U.S. Patent 4605 956, Aug. 12, 1986.

[26] K. Parulski and K. E. Spaulding, "Color image processing for digital cameras," in Digital Color Imaging Handbook, G. Sharma, Ed. Boca Raton, FL: CRC Press, 2002, pp. 728-757.

[27] R. Lukac and K. N. Plataniotis, "Normalized color-ratio modeling for CFA interpolation," IEEE Trans. Consumer Electron., vol. 50, no. 2, pp. 737-745, May 2004.

[28] N. Herodotou and A. N. Venetsanopoulos, "Color image interpolation for high resolution acquisition and display devices," IEEE Trans. Consumer Electron., vol. 41, no. 4, pp. 1118-1126, Nov. 1995.

[29] A. M. Darwish, M. S. Bedair, and S. I. Shaheen, "Adaptive resampling algorithm for image zooming," Proc. IEE-Vision, Image, Signal Processing, vol. 144, no. 4, pp. 207-212, Aug. 1997.

[30] S. Battiato, M. Guarnera, M. Mancuso, and A. Bruna, "Bayer image enlargement using correlated color components," in Proc. Int. Conf. Consumer Electronics, Jun. 2002, pp. 230-231.

[31] P. Longere, X. Zhang, P. B. Delahunt, and D. H. Brainard, "Perceptual assessment of demosaicing algorithm performance," Proc. IEEE, vol. 90, no. 1, pp. 123-132, Jan. 2002.

[32] D. R. Cok, "Signal Processing Method and Apparatus for Producing Interpolated Chrominance Values in a Sampled Color Image Signal," US Patent 4642 678, Feb. 10, 1987
[33] J. Adams, "Design of practical color filter array interpolation algorithms for digital cameras," in Proc. SPIE, vol. 3028, Feb. 1997, pp. 117-125.

[34] R. R. Schultz and R. L. Stevenson, "A Bayessian approach to image expansion for improved definition," IEEE Trans. Acoust., Speech Image Process., vol. 3, no. 3, pp. 233-242, May 1994.

[35] L. S. DeBruner and V. DeBrunner, "Color image interpolation with edgeenhancement," in Proc. 34th Asilomar Conf. Signals, Systems and Computers, vol. 2, Oct. 2000, pp. 901-905.

[36] K. Jenseen and D. Anastasiou, "Subpixel edge localization and interpolation of still images," IEEE Trans. Image Process., vol. 4, no. 3, pp. 285-295, Mar. 1995

[37] S. Thurnhofer and S. K. Mitra, "Edge-enhanced image zooming," Opt Eng., vol. 35, no. 7, pp. 1862-1869, Jul. 1996.

[38] L. Khriji and M. Gabbouj, "Directional-vector rational filters for color image interpolation," in Proc. 10th Int. Conf. Microelectronics, Dec. 1998, pp. 236-240.

[39] I. Pitas and A. N. Venetsanopoulos, "Order statistics in digital image processing," Proc. IEEE, vol. 80, no. 12, pp. 1892-1919, Dec. 1992.

[40] R. Ramanath, W. E. Snyder, G. L. Bilbro, and W. A. Sander, "Demosaicking methods for the Bayer color array," J. Electron. Imaging, vol. 11, no. 3, pp. 306-315, Jul. 2002.

[41] R. Lukac, K. N. Plataniotis, B. Smolka, and D. Hatzinakos, "A new color image zooming approach for digital still cameras," in Proc. 2nd Eur. Conf. Color in Graphics, Imaging and Vision, Apr. 2004, pp. 515-519.

[42] R. Lukac and K. N. Plataniotis, "A new digital camera zooming solution," in Proc. 17th Annu. Can. Conf. Electrical and Computer Engineering, vol. 2, May 2004, pp. 845-848.

[43] _ "Bayer pattern based digital zooming approach," in Proc. Int Symp. Circuits and Systems, vol. III, May 2004, pp. 253-256.

[44] K. N. Plataniotis, D. Androutsos, and A. N. Venetsanopoulos, "Adaptive fuzzy systems for multichannel signal processing," Proc. IEEE, vol. 87, no. 9, pp. 1601-1622, Sep. 1999.

[45] T. Sakamoto, C. Nakanishi, and T. Hase, "Software pixel interpolation for digital still cameras suitable for a 32-bit MCU," IEEE Trans. Consumer Electron., vol. 44, no. 4, pp. 1342-1352, Nov. 1998.

[46] J. W. Hwang and H. S. Lee, "Adaptive image interpolation based on local gradient features," IEEE Signal Process. Lett., vol. 11, no. 3, pp. 359-362, Mar. 2004.

[47] R. G. Keys, "Cubic convolution interpolation for digital image processing," IEEE Trans. Acoust., Speech Signal Process., vol. 29, no. 6 , pp. 1153-1160, Dec. 1981.

[48] S. E. Reichenbach and F. Geng, "Two-dimensional cubic convolution," IEEE Trans. Image Process., vol. 12, no. 8, pp. 857-865, Aug. 2003.

[49] G. Sharma and H. J. Trussell, "Digital color imaging," IEEE Trans. Image Process., vol. 6, no. 7, pp. 901-932, Jul. 1997.

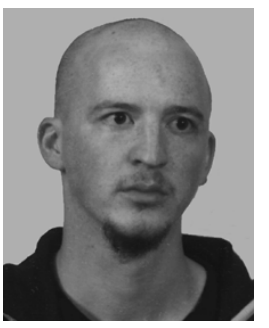

Rastislav Lukac (M'02) received the M.S. (Ing.) and $\mathrm{Ph} . \mathrm{D}$. degrees in telecommunications from the Technical University of Kosice, Kosice, Slovak Republic, in 1998 and 2001, respectively.

From February 2001 to August 2002, he was an Assistant Professor with the Department of Electronics and Multimedia Communications, Technical University of Kosice. During August 2002 to July 2003, he was a Researcher with the Slovak Image Processing Center, Dobsina, Slovak Republic. From January 2003 to March 2003, he was a Postdoctoral Fellow with the Artificial Intelligence and Information Analysis Laboratory, Aristotle University of Thessaloniki, Thessaloniki, Greece. Since May 2003, he has been a Postdoctoral Fellow with the Edward S. Rogers, Sr., Department of Electrical and Computer Engineering, University of Toronto, Toronto, ON, Canada. His research interests include digital camera image processing, microarray image processing, multimedia security, and nonlinear filtering and analysis techniques for color image and video processing.

Dr. Lukac is a Member of the IEEE Circuits and Systems, IEEE Consumer Electronics, and IEEE Signal Processing societies. He serves as a Technical Reviewer for various scientific journals and he participates as a Member of numerous international conference committees. In 2003, he was the recipient of the NATO/NSERC Science Award. 


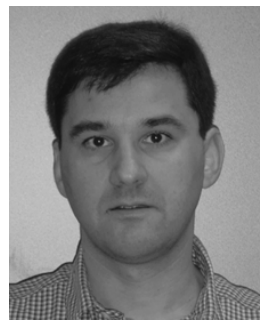

Konstantinos N. Plataniotis (S'90-M'92-SM'03) received the B.Eng. degree in computer engineering from the University of Patras, Patras, Greece, in 1988 and the M.S. and Ph.D. degrees in electrical engineering from the Florida Institute of Technology (Florida Tech), Melbourne, in 1992 and 1994, respectively.

He was affiliated with the Computer Technology Institute (CTI), Patras, from 1989 to 1991. From August 1997 to June 1999, he was an Assistant Professor with the School of Computer Science, Ryerson University, Toronto, ON, Canada. Since July 1999, he has been with the Edward S. Rogers, Sr. Department of Electrical and Computer Engineering, University of Toronto, Toronto, ON, Canada, where is currently an Associate Professor. He coauthored, with A. N. Venetsanopoulos, the book Color Image Processing \& Applications (Berlin, Germany: Springer-Verlag, May 2000), he is a contributor to seven books, and he has published more than 200 papers in refereed journals and conference proceedings in the areas of multimedia signal processing, image processing, adaptive systems, communications systems, and stochastic estimation.

Dr. Plataniotis is a past member of the IEEE Technical Committee on Neural Networks for Signal Processing and the chair of the IEEE Toronto Section. He was the Technical Co-Chair of the IEEE Canadian Conference on Electrical and Computer Engineering (CCECE) in 2001 and 2004. He is the Vice-chair of the 2006 Intelligent Transportation Systems Conference, and the Technical Program Co-chair for the IEEE 2006 International Conference in Multimedia and Expo (ICME 2006).

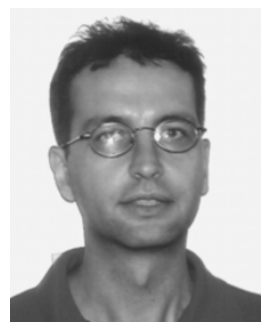

Dimitrios Hatzinakos (M'90-SM'98) received the Diploma degree from the University of Thessaloniki, Thessaloniki, Greece, in 1983, the M.A.Sc. degree from the University of Ottawa, Ottawa, ON, Canada, in 1986, and the Ph.D. degree from Northeastern University, Boston, MA, in 1990, all in electrical engineering.

In September 1990, he joined the Edward S. Rogers, Sr. Department of Electrical and Computer Engineering, University of Toronto, Toronto, ON, Canada, where now he holds the rank of Professor with tenure. Also, he served as Chair of the Communications Group of the Department during the period July 1999 to June 2004. Since November 2004, he has been the holder of the Bell Canada Chair in Mutimedia at the University of Toronto. His research interests are in the areas of multimedia signal processing and communications. He is the author/coauthor of more than 150 papers in technical journals and conference proceedings, and he has contributed to eight books in his areas of interest. His experience includes consulting through Electrical Engineering Consociates, Ltd., and contracts with United Signals and Systems, Inc., Burns and Fry, Ltd., Pipetronix, Ltd., Defense Research Establishment Ottawa (DREO), Vaytek, Inc., Nortel Networks, Vivosonic, Inc., and CANAMET, Inc.

Prof. Hatzinakos is a member of EURASIP, the Professional Engineers of Ontario (PEO), and the Technical Chamber of Greece. He served as an Associate Editor for the IEEE TRANSACTIONS ON SIGNAL PROCESSING from 1998 till 2002 and was Guest Editor for the special issue of Signal Processing on Signal Processing Technologies for Short Burst Wireless Communications, which appeared in October 2000. He was a member of the IEEE Statistical Signal and Array Processing Technical Committee (SSAP) from 1992 till 1995 and Technical Program Co-Chair of the 5th Workshop on Higher-Order Statistics in July 1997. 